The Existence of Weak Solutions to Single Porosity and Simple Dual-porosity Models of Two-phase Incompressible Flow

Todd Arbogast

February 1992

TR92-04 



\title{
THE EXISTENCE OF WEAK SOLUTIONS TO SINGLE POROSITY AND SIMPLE DUAL-POROSITY MODELS OF TWO-PHASE INCOMPRESSIBLE FLOW*†
}

\author{
TODD ARBogast \\ Department of Mathematical Sciences, \\ Rice University, Houston, Texas 77251-1892
}

Key words and phrases: porous medium, dual-porosity, two-phase, elliptic-parabolic, integro-differential, degenerate

Abstract. It is shown that there exists a weak solution to a degenerate and singular elliptic-parabolic partial integro-differential system of equations. These equations model two-phase incompressible flow of immiscible fluids in either an ordinary porous medium or in a naturally fractured porous medium. The full model is of dual-porosity type, though the single porosity case is covered by setting the matrixto-fracture flow terms to zero. This matrix-to-fracture flow is modeled simply in terms of fracture quantities; that is, no distinct matrix equations arise. The equations are considered in a global pressure formulation that is justified by appealing to a physical relation between the degeneracy of the wetting fluid's mobility and the singularity of the capillary pressure function. In this formulation, the elliptic and parabolic parts of the system are separated; hence, it is natural to consider various boundary conditions, including mixed nonhomogeneous, saturation dependent ones of the first three types. A weak solution is obtained as a limit of solutions to discrete time problems. The proof makes no use of the corresponding regularized system. The hypotheses required for various earlier results on single-porosity systems are weakened so that only physically relevant assumptions are made. In particular, the results cover the cases of a singular capillary pressure function, a pure Neumann boundary condition, and an arbitrary initial condition.

\section{INTRODUCTION}

The existence of weak solutions to the equations describing the flow of two incompressible, immiscible fluids in an unfractured, single porosity porous medium

\footnotetext{
${ }^{*}$ This work was supported by the National Science Foundation under grant DMS-8905505 and completed while the author was visiting both the Institute for Mathematics and its Applications, University of Minnesota, Minneapolis, Minnesota, and the University of Houston, Houston, Texas. $\nmid$ To appear in Journal of Nonlinear Analysis: Theory, Methods, and Applications
} 
has been shown in various ways [1], [4], [10], [11], [21], [22]. In this paper we improve upon these earlier results; moreover, we consider the question of existence for such flows in naturally fractured porous media in which the concept of dual-porosity [7], [28] is used to model the flow.

A naturally fractured reservoir is one that has throughout its extent an interconnected system of fractures that forms a porous structure distinct from that of the matrix (i.e., the porous rock). In a dual-porosity model, the transport of fluids is assumed to occur primarily within the fracture system, and this system is coupled to the matrix through locally defined macroscopic matrix source terms, one for each phase, that represent matrix-to-fracture flow. In general, a set of equations arises to describe the flow within each matrix block (i.e., each block of porous rock completely surrounded by fractures) [6], [14], [15], [16], [18], [20]; essentially, the local matrix-to-fracture flow is defined to be the net flow out of a matrix block. However, for some purposes a simplified model is considered in which the matrix source flow to the fracture is approximated by an explicit combination of the fracture quantities, and the matrix flow equations are neglected (see, e.g., [5], [12], [14], [15], [17], and [27]). Herein we show the existence of weak solutions to this type of model. In the process, we improve on the published results for ordinary single-porosity porous media flow since the unfractured case is treated by setting the matrix-to-fracture flow to zero.

We describe now the integro-differential system of equations considered in this paper. It is identical to the equations describing flow in unfractured porous media except for the introduction of the matrix source operator terms. Let $\Omega \subset \mathbb{R}^{d}$ and $I=(0, T], T>0$, denote the reservoir domain and the time interval of interest, respectively, and set $\Omega_{T}=\Omega \times I$. The usual unknowns are the wetting fluid "w" (water) phase saturation $s_{\mathrm{w}}=s(x, t)$ (rescaled to lie between zero and one so that the irreducible, or residual, saturations can henceforth be construed to be zero), the w-phase pressure $p_{\mathrm{w}}(x, t)$, and the nonwetting "o" (oil) phase pressure $p_{\mathrm{o}}(x, t)$. In terms of these variables, we have the following three equations:

$$
\begin{aligned}
& \phi \partial_{t} s-\nabla \cdot\left(\mathbf{k} \lambda_{\mathrm{w}}(s)\left(\nabla p_{\mathrm{w}}+\gamma_{\mathbf{w}}\right)\right)=f_{\mathrm{w}}(s)-F(s) \quad \text { in } \Omega_{T}, \\
& -\phi \partial_{t} s-\nabla \cdot\left(\mathbf{k} \lambda_{\mathrm{o}}(s)\left(\nabla p_{\mathrm{o}}+\gamma_{\mathrm{o}}\right)\right)=f_{\mathrm{o}}(s)+F(s) \\
& p_{\mathrm{c}}(s)=p_{\mathrm{o}}-p_{\mathrm{w}} \quad \text { in } \Omega_{T},
\end{aligned}
$$

where $\phi(x)$ and $\mathbf{k}(x)$ are the porosity and tensor of absolute permeability of the system of fractures, and for the phase $\theta=\mathrm{w}$ or $\mathrm{o}, \gamma_{\theta}(x)$ is the gravity-density vector term, $\lambda_{\theta}(x, s)$ is the phase mobility (i.e., the relative permeability divided by the phase viscosity), $f_{\theta}(x, t, s)$ is the distributed external volumetric $\theta$-source term, $p_{\mathrm{c}}(x, s)$ is the empirically given capillary pressure function, and $-F(x, t, s)$ is the macroscopically distributed matrix w-source operator, defined below (by incompressibility, $F(s)$ is the matrix o-source operator). Note that mathematically speaking, (1.3) defines $s(x, t)=S\left(x, p_{\mathrm{o}}-p_{\mathrm{w}}\right)$ as a function of $x$ and $p_{\mathrm{o}}-p_{\mathrm{w}}$. For 
the relevant physics, see, e.g., [8], [11], [13], [25], and [26] as general porous media references, and [7], [14], and [28] as dual-porosity references.

We consider a general integro-differential form for the matrix source operator. Let $\omega(x, t)$ be a nonnegative function that decays in time for each fixed $x \in \Omega$. For $\hat{\phi}(x) \geq 0$ and $\delta(t)$ the Dirac delta generalized function, define

$$
\begin{aligned}
F(x, t, s) & =\int_{0}^{t} \partial_{t} \sigma(x, s(x, t-\tau))[\hat{\phi}(x) \delta(\tau)+\omega(x, \tau)] d \tau \\
& =\hat{\phi}(x) \partial_{t} \sigma(x, s(x, t))+\int_{0}^{t} \partial_{t} \sigma(x, s(x, t-\tau)) \omega(x, \tau) d \tau
\end{aligned}
$$

where for each $x \in \Omega, \sigma(x, \cdot):[0,1] \rightarrow[0,1]$ is some increasing function that relates fracture system saturations to matrix saturations. To be more exact, if $p_{\mathrm{c}, \mathrm{m}}$ is the matrix capillary pressure function, then $\sigma(s)=p_{\mathrm{c}, \mathrm{m}}^{-1}\left(p_{\mathrm{c}}(s)\right)$ so that the matrix and fracture systems are in pressure equilibrium; see [6], [14], and [15]. The definition (1.4) is essentially what results from linearizing the equations describing matrix flow in the full model [5].

The general form (1.4) is able to handle two distinct types of simplified dualporosity models. Let $\phi_{\mathrm{m}}(x)$ denote the matrix porosity. If we take $\hat{\phi}=\phi_{\mathrm{m}}$ and $\omega(x, t) \equiv 0$, then

$$
F(x, t, s)=\phi_{\mathrm{m}}(x) \partial_{t} \sigma(x, s(x, t))
$$

and we have the "limit model" of [14], [15], and [17]. If we take instead $\hat{\phi} \equiv 0$ and

$$
\omega(x, t)=\phi_{\mathrm{m}}(x) \Lambda(x) e^{-\Lambda(x) t}
$$

for some positive rate constant $\Lambda(x)$, then

$$
\begin{aligned}
F(x, t, s)= & \phi_{\mathrm{m}}(x) \Lambda(x) \int_{0}^{t} \partial_{t} \sigma(x, s(x, t-\tau)) e^{-\Lambda(x) \tau} d \tau \\
=-\phi_{\mathrm{m}}(x) \Lambda(x)\{\Lambda(x) & \int_{0}^{t} \sigma(x, s(x, t-\tau)) e^{-\Lambda(x) \tau} d \tau \\
& \left.+\sigma(x, s(x, 0)) e^{-\Lambda(x) t}-\sigma(x, s(x, t))\right\}
\end{aligned}
$$

and we have the model of [5]. If also $\sigma$ is a multiple of the identity, we have the model of [12] and [27]. In the case of $(1.4 \mathrm{~b})$, note that $F(s)$ is not a differential operator since we can integrate by parts. In the general case, $\omega$ may be singular at $t=0$.

The most commonly encountered boundary conditions for underground reservoir problems are of Dirichlet, Neumann, and "third" type [8]; more generally, the latter 
two are in a saturation dependent form. We will consider these as well as the special "well" type boundary condition [11]. Decompose $\partial \Omega$ into three disjoint regions $\Gamma^{i}$, $i=1,2,3$, let $\Gamma^{3}=\bigcup_{j} \Gamma^{3, j}$ where each $\Gamma^{3, j}$ is connected, and set $\Gamma_{T}^{i(, j)}=\Gamma^{i(, j)} \times I$. We impose for $\theta=\mathrm{w}, \mathrm{o}$,

$$
\begin{aligned}
& p_{\theta}=p_{\theta}^{\mathrm{D}} \quad \text { on } \Gamma_{T}^{1}, \\
& -\mathbf{k} \lambda_{\theta}(s)\left(\nabla p_{\theta}+\gamma_{\theta}\right) \cdot \nu-a_{\theta}(s) p_{\theta}=g_{\theta}(s) \quad \text { on } \Gamma_{T}^{2}, \\
& \left.\begin{array}{l}
-\int_{\Gamma^{3, j}} \mathbf{k}\left(\lambda_{\mathrm{w}}(s)\left(\nabla p_{\mathrm{w}}+\gamma_{\mathrm{w}}\right)+\lambda_{\mathrm{o}}(s)\left(\nabla p_{\mathrm{o}}+\gamma_{\mathrm{o}}\right)\right) \cdot \nu=g_{j}^{\mathrm{W}} \\
p_{\theta}=p_{\theta}^{\mathrm{D}}+\pi_{j}^{\mathrm{W}}
\end{array}\right\} \text { on } \Gamma_{T}^{3, j},
\end{aligned}
$$

where $p_{\theta}^{\mathrm{D}}(x, t), a_{\theta}(x, t, s), g_{\theta}(x, t, s)$, and $g_{j}^{\mathrm{W}}(t)$ are given functions, $\pi_{j}^{\mathrm{W}}(t)$ are arbitrary scaling constants, and $\nu$ is the outer unit normal to $\partial \Omega$. Physically, $\Gamma^{1}$ represents the part of the boundary that is in contact with a liquid continuum. A prescribed flow rate is assumed over $\Gamma^{2}$ through a pervious boundary if $a_{\theta}=0$, through a semipervious boundary if $a_{\theta}>0$, and as a special but very important case through an impervious boundary if $a_{\theta}=0$ and $g_{\theta}=0$. The flow rate is generally assumed to be in the form of WOR, given water/oil production ratio, in which the flow rate of each phase is jointly proportional to its mobility and the total flow rate. Each $\Gamma^{3, j}$ represents the surface of a well through which the total flow rate is controlled, and on which a pressure distribution (but not its absolute scale) for each phase is maintained due to gravity and other factors.

The system is completed with a single initial condition for some $s^{0}(x)$ :

$$
s(\cdot, 0)=s^{0} \quad \text { on } \Omega .
$$

These equations are complicated primarily by three factors. First, the phase mobilities are degenerate; that is, at the irreducible $\mathrm{w}$ and o saturations (where our saturation is 0 and 1 , respectively), $\lambda_{\mathrm{w}}(0)=0$ and $\lambda_{\mathrm{o}}(1)=0$ (see Figure 1 for typical graphs of these functions). At these saturations one of the two phases ceases to flow. Second, $p_{\mathrm{c}}$ is a singular function of saturation: $p_{\mathrm{c}}(0)=+\infty$ is the usual case (see Figure 2). Third, $F(s)$ is in general an integro-differential delay operator of $s$.

The equations of two-phase incompressible flow in the single-porosity case (i.e., for $F(s) \equiv 0$ ) have been studied by Antoncev and Monahov [4], Chavent [10] and Chavent and Jaffré [11], Kružkov and Sukorjanskiı̌ [22], Kroener and Luckhaus [21], and Alt and di Benedetto [1]. Each of these authors showed the existence of a weak solution to the system with various boundary conditions under various hypotheses on the data.

Kružkov and Sukorjanskiı [22] assume nondegeneracy of the phase mobilities and nonsingularity of the capillary pressure. They then show the existence and uniqueness of classical solutions to the single porosity system. 


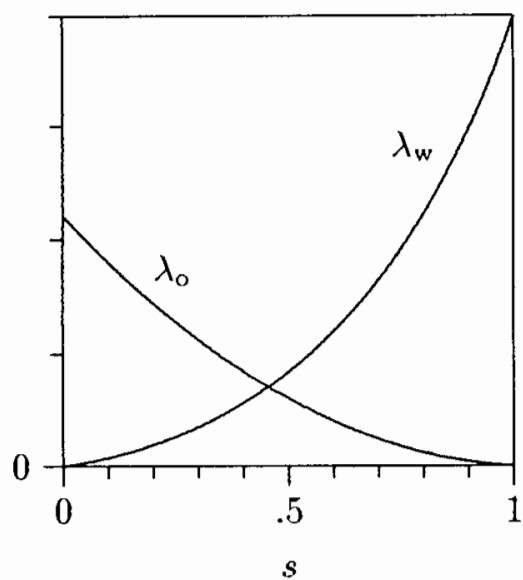

FIG. 1. Typical relative mobility functions.

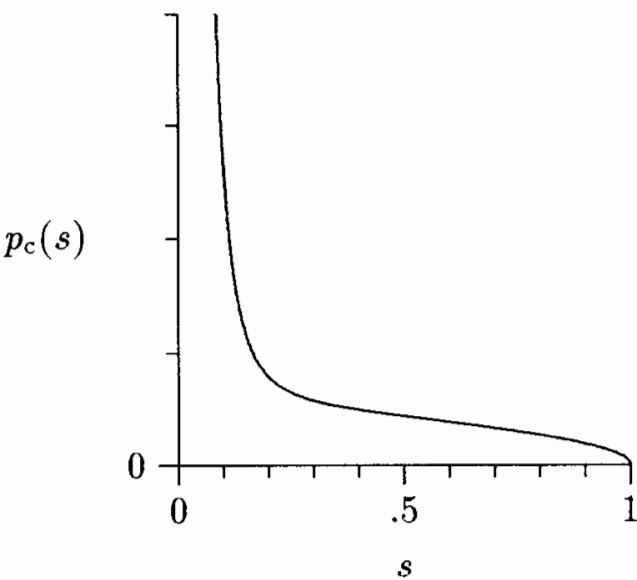

FIG. 2. A typical capillary pressure function (for fixed $x \in \Omega$ ).

Kroener and Luckhaus [21] require initial and boundary data that ensure the saturation remains bounded away from zero [21; Lemma 2.5] to obtain any results; hence, there is no $p_{c}$-singularity to contend with. In fact, to obtain a genuine weak solution, they require also that the saturation be bounded below one, thereby removing all degeneracy. Their results are based on a theorem of Alt and Luckhaus [2] giving the existence of weak solutions to general elliptic-parabolic systems. They extract their solution as a limit of solutions to certain regularized problems.

Antoncev and Monahov have shown many results concerning the single porosity equations and the corresponding nondegenerate regularized equations; a summary of their results appears in [4]. They use the mathematical concept of "global pressure" (see (2.1) below) to reformulate (1.1)-(1.3) as an elliptic equation coupled to a degenerate parabolic equation. Unfortunately, "third" and "well" type boundary conditions are not treated (i.e., $a_{\mathrm{w}} \equiv a_{\mathrm{o}} \equiv 0$ and $\Gamma^{3}=\emptyset$ ). Not all of their hypotheses reflect physically relevant constraints. The complication of a singular capillary pressure function is not considered because it is assumed that $0<c \leq\left|\partial p_{\mathrm{c}} / \partial s\right| \leq C<\infty$. Another major assumption is that $S\left(p_{\mathrm{o}}^{\mathrm{D}}-p_{\mathrm{w}}^{\mathrm{D}}\right)$ has space and time derivatives in $L^{2}\left(\Omega_{T}\right)$; hence, $\nabla\left(p_{\mathrm{o}}^{\mathrm{D}}-p_{\mathrm{w}}^{\mathrm{D}}\right) \in L^{2}\left(\Omega_{T}\right)$. This assumption on the Dirichlet data is stronger than what is formally obtained for the solution, that $\sqrt{\lambda_{\theta}(s)} \nabla p_{\theta} \in L^{2}\left(\Omega_{T}\right)$. A consistent approach would require the same smoothness for the data as is shown for the solution.

Chavent [10] and Chavent and Jaffré [11] do not consider the underground reservoir problem considered in this paper, since they impose boundary conditions that are appropriate for a water dam problem. Again in this work, some of their hypotheses are not physically relevant. Most seriously, Chavent and Jaffré require that $\left|p_{\mathrm{c}}\right|$ be uniformly bounded, removing one of the natural complications inherent in the system. For technical reasons they also require the unnatural condition that $\nabla s^{0} \in L^{2}(\Omega)$. They consider the system in a global pressure form. Their results 
are derived with the aid of Kakutani's fixed point theorem applied to certain penalized problems, and the solution is obtained as a limit of solutions to regularized problems.

Finally, Alt and di Benedetto [1] have considered the fully degenerate and singular single porosity system, though they limit themselves to problems with $\Gamma^{1} \neq \emptyset$, homogeneous Neumann conditions on $\Gamma^{2}$, and $\Gamma^{3}=\emptyset$ (though they admit "overflow" boundary conditions as well). However, like the above authors, not all of their hypotheses reflect physically relevant constraints. First, they require of the initial condition that

$$
\int_{0}^{p_{\mathrm{c}}\left(x, s^{0}\right)}\left(s^{0}-S(x, \xi)\right) d \xi \in L^{1}(\Omega),
$$

which restricts the blow-up of $p_{c}$ as $s \rightarrow 0^{+}$if $s^{0}$ is much near zero, the usual case for an oil-rich reservoir. Second, they require the not physically motivated condition that the $p_{\theta}^{\mathrm{D}} \in L^{\infty}\left(\Omega_{T}\right)$. (For example, if the reservoir were in contact with a lake, $p_{\mathrm{w}}^{\mathrm{D}}=-\infty$ on $\Gamma^{1}$.) Third, they require that $\nabla p_{\theta}^{\mathrm{D}} \in L^{2}\left(\Omega_{T}\right)$, even though they prove that the solution formally satisfies the weaker result $\sqrt{\lambda_{\theta}(s)} \nabla p_{\theta} \in L^{2}\left(\Omega_{T}\right)$. Alt and di Benedetto obtain some very important regularity results by making use of the global pressure formulation of the problem. However, their existence results use the techniques of [2] applied to regularized problems, so they do not treat the boundary conditions by considering separately the elliptic and parabolic parts of the differential system. As a consequence, they were unable to handle the important pure Neumann problem because it has an ambiguity in the pressure scale.

As far as the dual-porosity system is concerned, previous to this work, only the "limit model" ( $F(s)$ defined by $(1.4 \mathrm{a})$ ) has been analyzed. Under the assumptions of nondegenerate mobilities, nonsingular capillary pressures, and constant porosities, existence, uniqueness, and regularity results have been shown [17].

We will show the existence of a weak solution to the full system in a global pressure formulation. Our formulation will use as variables the global pressure $p$ and, rather than the saturation $s$, a "complementary pressure" $q$ (see (2.2) below). This transformation is achieved by noting a physical relation between the mobility of the wetting phase and the capillary pressure function, namely, that the quantity $\lambda_{\mathrm{w}} \partial p_{\mathrm{c}} / \partial s$ is integrable in $s$. This formulation is a natural setting for considering the boundary conditions (1.5)-(1.7). (It should also be a convenient setting for considering other specialized boundary conditions.) We will be able to weaken various assumptions made by the previously mentioned authors who obtained a portion of the results derived in this paper. We require the same amount of smoothness for the Dirichlet data as we show for the solution, and all of our assumptions are physically reasonable. The techniques we use are similar to those used by Alt, di Benedetto, and Luckhaus [1], [2]. One advantage of our formulation is that the system formally appears to be nondegenerate (see (2.5)-(2.7) below). As a consequence, we need not extract our solution as the limit of solutions to regularized or penalized problems; 
we obtain it more directly.

\section{The Global Pressure Formulation}

Let us begin this section by stating the following assumptions, some of which are more general than is needed for the physical problem. (In this paper, $H^{k}=W^{k, 2}$ where $W^{k, r}$ is the set of functions in the Lebesgue space $L^{r}$ with derivatives up to order $k$ in $L^{r}$.)

(A1) Let $\Omega \subset \mathbb{R}^{d}$ be a bounded, connected domain with Lipschitz boundary. Let $\partial \Omega=\bigcup_{i=1}^{3} \overline{\Gamma_{\mathrm{p}}^{i}}=\bigcup_{i=1}^{2} \overline{\Gamma_{\mathrm{s}}^{i}}, \Gamma_{\mathrm{p}}^{i} \cap \Gamma_{\mathrm{p}}^{j}=\emptyset$ for $i \neq j$ and $\Gamma_{\mathrm{s}}^{1} \cap \Gamma_{\mathrm{s}}^{2}=\emptyset$, where each $\Gamma_{\mathrm{p}}^{i}$ and $\Gamma_{\mathrm{s}}^{i}$ is a $(d-1)$-dimensional domain. Further, let $\Gamma_{\mathrm{p}}^{3}=\bigcup_{j} \Gamma_{\mathrm{p}}^{3, j}$, where the $\Gamma_{\mathrm{p}}^{3, j}$ are the connectivity components of $\Gamma_{\mathrm{p}}^{3}$. Finally, suppose that $\overline{\Gamma_{\mathrm{p}}^{1}} \cap \overline{\Gamma_{\mathrm{p}}^{3}}=\emptyset$ and $\Gamma_{\mathrm{p}}^{1} \cup \Gamma_{\mathrm{p}}^{3} \subset \Gamma_{\mathrm{s}}^{1}$. (Of course we will write $\Gamma_{\mathrm{p}, T}^{i(, j)}=\Gamma_{\mathrm{p}}^{i(, j)} \times I$ and $\Gamma_{\mathrm{s}, T}^{i}=\Gamma_{\mathrm{s}}^{i} \times I$.)

(A2) Let $\phi \in L^{\infty}(\Omega)$ and $\phi(x) \geq \phi_{*}>0$.

(A3) Let $\mathbf{k}(x)$ be a bounded, measurable, uniformly positive definite symmetric tensor on $\Omega$ (i.e., for any nonzero $\xi \in \mathbb{R}^{d}, 0<k_{*} \leq|\xi|^{-2} \sum_{i, j} k_{i j}(x) \xi_{i} \xi_{j} \leq$ $\left.k^{*}<\infty\right)$.

(A4) The $\lambda_{\theta}(x, s)$ are measurable in $x \in \Omega$ and continuous in $s \in[0,1]$, and have graphs as depicted in Figure 1: $\lambda_{\mathbf{w}}(0)=0, \lambda_{\mathbf{w}}(s)>0$ for $s>0, \lambda_{\mathbf{o}}(1)=0$, and $\lambda_{0}(s)>0$ for $s<1$. Let $\lambda(x, s)=\lambda_{w}(x, s)+\lambda_{0}(x, s)$ and assume that $0<\lambda_{*} \leq \lambda(x, s) \leq \lambda^{*}<\infty$.

$\left(\mathrm{A} 5 \mathrm{a}^{*}\right)$ The $\lambda_{\theta}(x, s)$ are differentiable in $x \in \Omega$.

$\left(\mathrm{A} 5 \mathrm{~b}^{*}\right)$ Suppose $p_{\mathrm{c}}(x, s)$ is strictly monotone decreasing in $s$ and is jointly differentiable in $x \in \Omega$ and locally Lipschitz continuous in $s \in(0,1]$.

$\left(\mathrm{A} 5 \mathrm{c}^{*}\right.$ ) Suppose that $p_{\mathrm{c}}$ and the $\lambda_{\theta}$ are related in such a way that

(i) $\int_{0}^{1}\left(\lambda_{\mathrm{w}} \frac{\partial p_{\mathrm{c}}}{\partial s}\right)(x, \varsigma) d \varsigma \in L^{2}(\Omega)$

(ii) $\int_{0}^{1}\left|\nabla_{x}\left(\frac{\lambda_{\mathrm{w}}}{\lambda} \frac{\partial p_{\mathrm{c}}}{\partial s}\right)(x, \varsigma)\right| d \varsigma+\int_{0}^{1}\left|\nabla_{x}\left(\frac{\lambda_{\mathrm{w}} \lambda_{\mathrm{o}}}{\lambda^{2}} \frac{\partial p_{\mathrm{c}}}{\partial s}\right)(x, \varsigma)\right| d \varsigma \in L^{2}(\Omega)$.

(iii) $\sup _{s \in[0,1]}\left|\left(\lambda_{\mathrm{w}} \nabla_{x} p_{\mathrm{c}}\right)(x, s)\right| \in L^{2}(\Omega)$.

We remark that (A5c*) is physically reasonable by Leverett's semi-empirical equation

$$
p_{\mathrm{c}}(x, s)=\kappa(x) J(s)
$$

(see [8], [23], [26]) and by Burdine's theoretical relationship between relative permeability and capillary pressure:

$$
\lambda_{\mathbf{w}}(s) \propto s^{2} \int_{0}^{s} \frac{d \varsigma}{J^{2}(\varsigma)}
$$


(see [8], [9], [26]). We need only assume that $|J(1)|<\infty$ and that $\kappa$ and the $\lambda_{\theta}$ are sufficiently smooth.

Our desire is to find a solution in some Sobolev space. It is important to choose a reasonable set of dependent variables, since it is easily seen that $p_{\mathrm{w}}=-\infty$ if $s=0$ (oil then being a continuous phase implies that $p_{\mathrm{o}}$ is well behaved). Hence $p_{\mathrm{w}}$ cannot in general be expected to lie in any Sobolev space. Because of $\left(\mathrm{A} 5 \mathrm{c}^{*}\right)$, we can define the global pressure ([3], [10]) as

$$
p=p_{\mathrm{o}}-\int_{0}^{s}\left(\frac{\lambda_{\mathrm{w}}}{\lambda} \frac{\partial p_{\mathrm{c}}}{\partial s}\right)(x, \varsigma) d \varsigma=p_{\mathrm{o}}+\int_{p_{\mathrm{o}}-p_{\mathrm{w}}}^{p_{\mathrm{c}}(x, 0)}\left(\frac{\lambda_{\mathrm{w}}}{\lambda}\right)(x, S(x, \xi)) d \xi,
$$

provided only that at each $x \in \Omega, p_{\mathrm{o}}-p_{\mathrm{w}}$ is indeed in the range of $p_{\mathrm{c}}$. We can also define a "complementary pressure"

$$
q=-\int_{0}^{s}\left(\frac{\lambda_{\mathrm{w}} \lambda_{\mathrm{o}}}{\lambda^{2}} \frac{\partial p_{\mathrm{c}}}{\partial s}\right)(x, \varsigma) d \varsigma=\int_{p_{\mathrm{o}}-p_{\mathrm{w}}}^{p_{\mathrm{c}}(x, 0)}\left(\frac{\lambda_{\mathrm{w}} \lambda_{\mathrm{o}}}{\lambda^{2}}\right)(x, S(x, \xi)) d \xi .
$$

Then with (1.3),

$$
\begin{aligned}
\lambda(s) \nabla p=\lambda_{\mathrm{w}} & (s) \nabla p_{\mathrm{w}}+\lambda_{\mathrm{o}}(s) \nabla p_{\mathrm{o}} \\
& +\left(\lambda_{\mathrm{w}} \nabla_{x} p_{\mathrm{c}}\right)(x, s)-\lambda(s) \int_{0}^{s} \nabla_{x}\left(\frac{\lambda_{\mathrm{w}}}{\lambda} \frac{\partial p_{\mathrm{c}}}{\partial s}\right)(x, \varsigma) d \varsigma \\
\lambda(s) \nabla q=- & \left(\frac{\lambda_{\mathrm{w}} \lambda_{\mathrm{o}}}{\lambda}\right)(s) \nabla\left(p_{\mathrm{o}}-p_{\mathrm{w}}\right) \\
& +\left(\frac{\lambda_{\mathrm{w}} \lambda_{\mathrm{o}}}{\lambda} \nabla_{x} p_{\mathrm{c}}\right)(x, s)-\lambda(s) \int_{0}^{s} \nabla_{x}\left(\frac{\lambda_{\mathrm{w}} \lambda_{\mathrm{o}}}{\lambda^{2}} \frac{\partial p_{\mathrm{c}}}{\partial s}\right)(x, \varsigma) d \varsigma .
\end{aligned}
$$

In terms of these variables, we obtain for the sum of (1.1) and (1.2), (1.1), and (1.3),

$$
\begin{aligned}
-\nabla \cdot\left[\mathbf{k}\left(\lambda(s) \nabla p+\gamma_{1}(s)\right)\right] & =f(s) & & \text { in } \Omega_{T}, \\
\phi \partial_{t} s-\nabla \cdot\left[\mathrm{k}\left(\lambda(s) \nabla q+\lambda_{\mathbf{w}}(s) \nabla p+\gamma_{2}(s)\right)\right] & =f_{\mathbf{w}}(s)-F(s) & & \text { in } \Omega_{T}, \\
s & =\mathcal{S}(q) & & \text { in } \Omega_{T},
\end{aligned}
$$

where

$$
\begin{aligned}
& \gamma_{1}(x, s)=-\left(\lambda_{\mathbf{w}} \nabla_{x} p_{\mathrm{c}}\right)(x, s)+\lambda(x, s) \int_{0}^{s} \nabla_{x}\left(\frac{\lambda_{\mathrm{w}}}{\lambda} \frac{\partial p_{\mathrm{c}}}{\partial s}\right)(x, \varsigma) d \varsigma \\
&+\lambda_{\mathbf{w}}(x, s) \gamma_{\mathrm{w}}(x)+\lambda_{\mathrm{o}}(x, s) \gamma_{\mathrm{o}}(x) \\
& \gamma_{2}(x, s)=-\left(\lambda_{\mathbf{w}} \nabla_{x} p_{\mathrm{c}}\right)(x, s)+\lambda(x, s) \int_{0}^{s} \nabla_{x}\left(\frac{\lambda_{\mathrm{w}} \lambda_{\mathrm{o}}}{\lambda^{2}} \frac{\partial p_{\mathrm{c}}}{\partial s}\right)(x, \varsigma) d \varsigma \\
&+\lambda_{\mathbf{w}}(x, s) \int_{0}^{s} \nabla_{x}\left(\frac{\lambda_{\mathrm{w}}}{\lambda} \frac{\partial p_{\mathrm{c}}}{\partial s}\right)(x, \varsigma) d \varsigma+\lambda_{\mathbf{w}}(x, s) \gamma_{\mathrm{w}}(x), \\
& f(x, t, s)=f_{\mathbf{w}}(x, t, s)+f_{\mathrm{o}}(x, t, s)
\end{aligned}
$$


and, with

$$
q^{*}(x)=-\int_{0}^{1}\left(\frac{\lambda_{\mathrm{w}} \lambda_{\mathrm{o}}}{\lambda^{2}} \frac{\partial p_{\mathrm{c}}}{\partial s}\right)(x, \varsigma) d \varsigma,
$$

$\mathcal{S}(x, q)=S\left(x, p_{\mathrm{o}}-p_{\mathrm{w}}\right)$ is the inverse of $(2.2)$ for $0 \leq q \leq q^{*}(x)$. The boundary conditions become (if $\Gamma_{\mathrm{p}}^{i(, j)}=\Gamma^{i(, j)}, \Gamma_{\mathrm{s}}^{2}=\Gamma^{2}$, and $\Gamma_{\mathrm{s}}^{1}=\Gamma^{1} \cup \Gamma^{3}$ )

$$
\begin{aligned}
& p=p^{\mathrm{D}} \quad \text { on } \Gamma_{\mathrm{p}, T}^{1}, \\
& -\mathbf{k}\left(\lambda(s) \nabla p+\gamma_{1}(s)\right) \cdot \nu-a(s) p=G(s) \quad \text { on } \Gamma_{\mathrm{p}, T}^{2}, \\
& \left.\begin{array}{l}
-\int_{\Gamma_{\mathrm{p}}^{3, j}} \mathbf{k}\left(\lambda(s) \nabla p+\gamma_{1}(s)\right) \cdot \nu=g_{j}^{\mathrm{W}} \\
p=p^{\mathrm{D}}+\pi_{j}^{\mathrm{W}}
\end{array}\right\} \text { on } \Gamma_{\mathrm{p}, T}^{3, j}, \\
& \left.\begin{array}{l}
q=q^{\mathrm{D}} \quad \text { on } \Gamma_{\mathbf{s}, T}^{1}, \\
-\mathbf{k}\left(\lambda(s) \nabla q+\lambda_{\mathbf{w}}(s) \nabla p+\gamma_{2}(s)\right) \cdot \nu-a_{\mathbf{w}}(s) p=G_{\mathbf{w}}(s) \quad \text { on } \Gamma_{\mathbf{s}, T}^{2},
\end{array}\right\}
\end{aligned}
$$

where $p^{\mathrm{D}}(x, t), q^{\mathrm{D}}(x, t)$ are the transforms of $p_{\mathrm{w}}^{\mathrm{D}}(x, t), p_{\mathrm{o}}^{\mathrm{D}}(x, t)$ by the latter expressions in (2.1)-(2.2),

$$
\begin{aligned}
a(x, t, s)= & a_{\mathbf{w}}(x, t, s)+a_{\mathrm{o}}(x, t, s) \\
G(x, t, s)= & g_{\mathbf{w}}(x, t, s)+g_{\mathrm{o}}(x, t, s) \\
& +a(x, t, s) \int_{0}^{s}\left(\frac{\lambda_{\mathrm{w}}}{\lambda} \frac{\partial p_{\mathrm{c}}}{\partial s}\right)(x, \varsigma) d \varsigma-a_{\mathrm{w}}(x, t, s) p_{\mathbf{c}}(x, s) \\
G_{\mathrm{w}}(x, t, s)=g_{\mathbf{w}}(x, t, s) & \\
& +a_{\mathrm{w}}(x, t, s) \int_{0}^{s}\left(\frac{\lambda_{\mathrm{w}}}{\lambda} \frac{\partial p_{\mathrm{c}}}{\partial s}\right)(x, \varsigma) d \varsigma-a_{\mathrm{w}}(x, t, s) p_{\mathrm{c}}(x, s) .
\end{aligned}
$$

Finally, (1.8) becomes

$$
q(\cdot, 0)=q^{0} \quad \text { on } \Omega,
$$

where $q^{0}(x)$ is the transform of $s^{0}(x)$ by $(2.2)$.

\section{Statement of the Main Result}

We require a bit of notation. Let

$$
\begin{aligned}
& V=\left\{v \in H^{1}(\Omega):\left.v\right|_{\Gamma_{\mathrm{p}}^{1}}=0,\left.v\right|_{\Gamma_{\mathrm{P}}^{3, j}} \text { is constant for each } j,\right. \\
& \text { and if } \left.\Gamma_{\mathrm{p}}^{1}=\emptyset \text { and } a \equiv 0, \text { then } \int_{\Omega} v d x=0\right\}, \\
& W=\left\{w \in H^{1}(\Omega):\left.w\right|_{\Gamma_{\mathrm{s}}^{1}}=0\right\},
\end{aligned}
$$


and let $V^{*}$ and $W^{*}$ be the duals of $V$ and $W$, respectively. Let $(\cdot, \cdot)_{R}$ denote the $L^{2}(R)$ inner product, and simplify the notation by writing $\|\cdot\|_{k, R}$ for $\|\cdot\|_{H^{k}(R)}$, where $R$ will be omitted if $R=\Omega$. For $\psi=\psi(x, s)$, let

$$
\|\psi\|\|=\| \sup _{s \in[0,1]}|\psi(x, s)| \|
$$

for any given norm.

We now state the rest of our assumptions, again more generally than is necessary for the physical problem. We begin by recasting $\left(\mathrm{A} 5 \mathrm{a}-\mathrm{c}^{*}\right)$.

(A5) Suppose that $0<q^{*} \in H^{1}(\Omega)$ and that $\mathcal{S}:\{(x, q): x \in \Omega$ and $0 \leq q \leq$ $\left.q^{*}(x)\right\} \rightarrow[0,1]$ is measurable in $x$, continuous and strictly monotone increasing in $q$, and satisfies $\mathcal{S}(x, 0)=0$ and $\mathcal{S}\left(x, q^{*}(x)\right)=1$.

(A6) Each of $\gamma_{1}, \gamma_{2}, f, f_{\mathrm{w}}, a, a_{\mathrm{w}}, G$, and $G_{\mathrm{w}}$ is continuous in $s$, and for some fixed $\rho \in[2, \infty]$, the following norms are bounded:

$$
\begin{array}{cccc}
\left\|\gamma_{1}\right\|_{L^{\rho}\left(I ; L^{2}(\Omega)\right)}, & \|f\|_{L^{\rho}\left(I ; V^{*}\right)}, & \|a\|_{L^{\infty}\left(\Omega_{T}\right)}, & \|G\|_{L^{\rho}\left(I ; H^{-1 / 2}\left(\Gamma_{\mathrm{p}}^{2}\right)\right)}, \\
\|\| \gamma_{2} \|_{L^{2}\left(I ; L^{2}(\Omega)\right)}, & \left\|f_{\mathrm{w}}\right\|_{L^{2}\left(I ; W^{*}\right)}, & \left\|a_{\mathrm{W}}\right\|_{L^{\infty}\left(\Omega_{T}\right)}, & \left\|G_{\mathrm{w}}\right\|_{L^{2}\left(I ; H^{-1 / 2}\left(\Gamma_{\mathrm{s}}^{2}\right)\right)}
\end{array}
$$

Moreover, $a \geq 0$ and $\left\{\sum_{j}\left(g_{j}^{\mathrm{W}}\right)^{2}\right\}^{1 / 2} \in L^{\rho}(I)$.

(A7) Suppose that:

(i) $\gamma_{2}(0)=0$ and $\gamma_{1}(1)-\gamma_{2}(1)=\lambda(1) \nabla q^{*}$ in $\Omega_{T}$;

(ii) $f_{\mathrm{w}}(0) \geq 0$ and $f_{\mathrm{o}}(1)=f(1)-f_{\mathrm{w}}(1) \geq 0$ in $\Omega_{T}$;

(iii) $a_{\mathrm{w}}(0)=0$ and $a_{\mathrm{o}}(1)=a(1)-a_{\mathrm{w}}(1)=0$ on $\Gamma_{\mathrm{s}, T}^{2}$.

(iv) $g_{\mathrm{w}}(0)=G_{\mathrm{w}}(0) \leq 0$ and $g_{\mathrm{o}}(1)=G(1)-G_{\mathrm{w}}(1) \leq 0$ on $\Gamma_{\mathrm{s}, T}^{2}$;

(A8) If $\Gamma_{\mathbf{p}}^{1}=\emptyset$ and $a \equiv 0$, then $f$ and $G$ are independent of $s$ and $\int_{\Gamma_{\mathrm{p}}^{2}} G(x, t) d A(x)+$ $\sum_{j} g_{j}^{\mathrm{W}}(t)=\int_{\Omega} f(x, t) d x$. There is some set $\Gamma_{\mathrm{p}}^{2, *} \subset \Gamma_{\mathrm{p}}^{2}$ (with nonzero measure only if $\Gamma_{\mathrm{p}}^{1}=\emptyset$ and $\left.a \not \equiv 0\right)$ such that for all $(x, t, s) \in \Gamma_{\mathrm{p}}^{2, *} \times I \times[0,1]$, $a(x, t, s) \geq a_{*}>0$.

(A9) Suppose $p^{\mathrm{D}} \in L^{\rho}\left(I ; H^{1}(\Omega)\right), q^{\mathrm{D}} \in L^{2}\left(I ; H^{1}(\Omega)\right), \partial_{t} q^{\mathrm{D}} \in L^{1}\left(\Omega_{T}\right)$, and $0 \leq$ $q^{\mathrm{D}}(x, t) \leq q^{*}(x)$ a.e. on $\Omega_{T}$.

(A10) Let $q^{0} \in L^{2}(\Omega)$ satisfy $0 \leq q^{0}(x) \leq q^{*}(x)$ a.e. on $\Omega$.

(A11) Let $\sigma(x, s)$ be measurable in $x$, continuous and strictly monotone increasing in $s$, and map onto $[0,1]$.

(A12) Let $\hat{\phi} \in L^{\infty}(\Omega)$ and $\hat{\phi} \geq 0$.

(A13) Suppose $\omega \in L^{1}\left(I ; L^{\infty}(\Omega)\right), \omega \geq 0$, and for each fixed $x \in \Omega, \omega(x, t)$ is monotone nonincreasing in $t \in I$.

We should comment on (A7). First, (A7i) reflects (2.8)-(2.9). Second, (A7iii) merely says that a semipermeable boundary is impermeable to the $\theta$-phase if this phase is absent. Finally, (A7ii, iv) reflect WOR-type conditions where external 
volumetric sources of injection (either from within $\Omega$ or on $\partial \Omega$ ) are unconstrained but sinks of production are proportional to phase mobility.

Note that formally we can write

$$
\begin{aligned}
F(x, t, s) & =\hat{\phi}(x) \partial_{t} \sigma(x, s(x, t))+\int_{0}^{t} \partial_{t} \sigma(x, s(x, t-\tau)) \omega(x, \tau) d \tau \\
& =\partial_{t}\left\{\hat{\phi}(x) \sigma(x, s(x, t))+\int_{0}^{t}\left[\sigma(x, s(x, t-\tau))-\sigma\left(x, s^{0}(x)\right)\right] \omega(x, \tau) d \tau\right\} \\
& =\partial_{t}\left[\hat{\phi} \sigma(s)+\left(\sigma(s)-\sigma\left(s^{0}\right)\right) * \omega\right](x, t),
\end{aligned}
$$

where "*" denotes convolution in time. With $\rho^{*}=\rho /(\rho-1)$, we can now define what is meant by a weak solution to the problem.

Definition. A weak solution to the system (2.5)-(2.7), (2.12)-(2.13), (2.17) is a pair of functions $p \in L^{\rho}(I ; V)+p^{\mathrm{D}}$ and $q \in L^{2}(I ; W)+q^{\mathrm{D}}$ such that

$$
\partial_{t}\left[\phi s+\hat{\phi} \sigma(s)+\left(\sigma(s)-\sigma\left(s^{0}\right)\right) * \omega\right] \in L^{2}\left(I ; W^{*}\right)
$$

and

$$
\begin{aligned}
& 0 \leq q(x, t) \leq q^{*}(x) \quad \text { almost everywhere on } \Omega_{T}, \\
& s=\mathcal{S}(q), \\
& \int_{I}\left(\mathbf{k}\left[\lambda(s) \nabla p+\gamma_{1}(s)\right], \nabla \xi\right)+\int_{I}(G(s)+a(s) p, \xi)_{\Gamma_{\mathrm{P}}^{2}}+\left.\sum_{j} \int_{I} g_{j}^{\mathrm{W}} \xi\right|_{\Gamma_{\mathrm{P}}^{3, j}} \\
& =\int_{I}(f(s), \xi) \quad \text { for all } \xi \in L^{\rho^{*}}(I ; V), \\
& \int_{I}\left\langle\partial_{t}\left[\phi s+\hat{\phi} \sigma(s)+\left(\sigma(s)-\sigma\left(s^{0}\right)\right) * \omega\right], \zeta\right\rangle \\
& +\int_{I}\left(\mathbf{k}\left[\lambda(s) \nabla q+\lambda_{\mathrm{w}}(s) \nabla p+\gamma_{2}(s)\right], \nabla \zeta\right) \\
& +\int_{I}\left(G_{\mathrm{w}}(s)+a_{\mathrm{w}}(s) p, \zeta\right)_{\Gamma_{s}^{2}} \\
& =\int_{I}\left(f_{\mathbf{w}}(s), \zeta\right) \quad \text { for all } \zeta \in L^{2}(I ; W),
\end{aligned}
$$

and finally

$$
\begin{aligned}
& \int_{I}\left\langle\partial_{t}[\phi s\right.\left.\left.+\hat{\phi} \sigma(s)+\left(\sigma(s)-\sigma\left(s^{0}\right)\right) * \omega\right], \zeta\right\rangle \\
&+\int_{I}\left(\phi\left(s-s^{0}\right)+\hat{\phi}\left(\sigma(s)-\sigma\left(s^{0}\right)\right)+\left(\sigma(s)-\sigma\left(s^{0}\right)\right) * \omega, \partial_{t} \zeta\right) \\
&=0 \quad \text { for all } \zeta \in L^{2}(I ; W) \cap W^{1,1}\left(I ; L^{1}(\Omega)\right) \text { with } \zeta(x, T)=0 .
\end{aligned}
$$


Here and throughout, $\left.s\right|_{\partial \Omega}$ should be interpreted as $\mathcal{S}\left(\left.q\right|_{\partial \Omega}\right)$.

In the next section we prove:

Theorem 1. Assume (A1)-(A13). Then there exists a weak solution to the system in the sense of the Definition.

REMARK: The assumption (A9) on the transformed functions $p^{\mathrm{D}}$ and $q^{\mathrm{D}}$ is consistent with our results for $p$ and $q$. In terms of the original variables (i.e., by $(2.3)-(2.4)$ and $\left.\left(A 5 c^{*}\right)\right),(A 9)$ requires that

$$
\lambda_{\theta}\left(S\left(p_{\mathrm{o}}^{\mathrm{D}}-p_{\mathrm{w}}^{\mathrm{D}}\right)\right) \nabla p_{\theta}^{\mathrm{D}} \in L^{2}\left(\Omega_{T}\right)
$$

this is consistent with what we obtain for the $p_{\theta}$, that $\lambda_{\theta}(s) \nabla p_{\theta} \in L^{2}\left(\Omega_{T}\right)$.

\section{Proof of Theorem 1}

Our method of proof uses the technique of [2] to deal with the time derivative of $s$; that is, we approximate it by a backward difference. For each positive integer $\tilde{N}$, partition $I$ into $N=2^{\tilde{N}}$ subintervals, each of length $h=T / N=2^{-\tilde{N}} T$. These partitions are nested for simplicity. Let $t_{n}=n h$ and $I_{n}=\left(t_{n-1}, t_{n}\right]$ for an integer $n$, and denote the time difference operator by

$$
\partial^{\eta} u(t)=\frac{u(t+\eta)-u(t)}{\eta}
$$

for any function $u(t)$ and any $\eta \in \mathbb{R}$. For $\mathcal{H}$ a Hilbert space, let

$$
\begin{aligned}
\ell_{h}(\mathcal{H})=\left\{v \in L^{\infty}(I ; \mathcal{H}):\right. \\
\left.\quad v \text { is piecewise constant in time on each subinterval } I_{n} \subset I\right\}
\end{aligned}
$$

For convenience, if $u \in \ell_{h}(\mathcal{H})$, let $u^{n}=\left.u\right|_{I_{n}}$ for $n \leq N$. We approximate for $t \in I_{n}$

$$
p_{h}^{\mathrm{D}}(x, t)=\frac{1}{h} \int_{I_{n}} p^{\mathrm{D}}(x, \tau) d \tau \quad \text { and } \quad q_{h}^{\mathrm{D}}(x, t)=\frac{1}{h} \int_{I_{n}} q^{\mathrm{D}}(x, \tau) d \tau
$$

Our approximation scheme is the following: Find $p_{h} \in \ell_{h}(V)+p_{h}^{\mathrm{D}}$ and $q_{h} \in$ 
$\ell_{h}(W)+q_{h}^{\mathrm{D}}$, extended such that $q_{h}=q^{0}$ for $t \leq 0$, satisfying

$$
\begin{aligned}
& s_{h}=\mathcal{S}\left(q_{h}\right), \\
& \int_{I}\left(\mathbf{k}\left[\lambda\left(s_{h}\right) \nabla p_{h}+\gamma_{1}\left(s_{h}\right)\right], \nabla \xi\right)+\int_{I}\left(G\left(s_{h}\right)+a\left(s_{h}\right) p_{h}, \xi\right)_{\Gamma_{\mathrm{p}}^{2}}+\left.\sum_{j} \int_{I} g_{j}^{\mathrm{W}} \xi\right|_{\Gamma_{\mathrm{p}}^{3, j}} \\
& =\int_{I}\left(f\left(s_{h}\right), \xi\right) \quad \text { for all } \xi \in \ell_{h}(V) \\
& \quad \int_{I}\left(\partial^{-h}\left[\phi s_{h}+\hat{\phi} \sigma\left(s_{h}\right)+\left(\sigma\left(s_{h}\right)-\sigma\left(s^{0}\right)\right) *_{h} \omega\right], \zeta\right) \\
& \quad+\int_{I}\left(\mathbf{k}\left[\lambda\left(s_{h}\right) \nabla q_{h}+\lambda_{\mathrm{w}}\left(s_{h}\right) \nabla p_{h}+\gamma_{2}\left(s_{h}\right)\right], \nabla \zeta\right) \\
& \quad+\int_{I}\left(G_{\mathrm{w}}\left(s_{h}\right)+a_{\mathrm{w}}\left(s_{h}\right) p_{h}, \zeta\right)_{\Gamma_{\mathrm{s}}^{2}} \\
& =\int_{I}\left(f_{\mathrm{w}}\left(s_{h}\right), \zeta\right) \quad \text { for all } \zeta \in \ell_{h}(W)
\end{aligned}
$$

For any $\zeta \in \ell_{h}(W)$, we mean by " $\zeta *_{h} \omega$ " that for $t \in I_{n}$,

$$
\begin{aligned}
\left(\zeta *_{h} \omega\right)(x, t) & =\int_{0}^{t_{n}} \zeta\left(x, t^{n}-\tau\right) \omega(x, \tau) d \tau \\
& =\sum_{k=1}^{n} \zeta^{n-k+1}(x) \int_{t_{k-1}}^{t_{k}} \omega(x, \tau) d \tau=\sum_{k=1}^{n} \zeta^{k}(x) \omega_{n-k}(x),
\end{aligned}
$$

where $\omega_{j}(x)=\int_{I_{j+1}} \omega(x, \tau) d \tau$. Note that for $t \in I_{n}$,

$$
\partial^{-h}\left(\sigma\left(s_{h}\right)-\sigma\left(s^{0}\right)\right) *_{h} \omega=\sum_{k=1}^{n}\left(\sigma\left(s_{h}^{k}\right)-\sigma\left(s_{h}^{k-1}\right)\right) \omega_{n-k} .
$$

The heart of our existence proof is contained in Lemmas 1-4 below. Throughout, $c$ and $C$ will denote generic positive constants, $c$ small and $C$ large, that are independent of $h$ unless such dependence is explicitly indicated.

LEMMA 1 . For any $h$ sufficiently small, there exists a solution to the approximation scheme (4.2)-(4.4); moreover,

$$
0 \leq q_{h}(x, t) \leq q^{*}(x) \text { a.e. on } \Omega_{T} .
$$

This is purely an elliptic result; to maintain the continuity of our argument, we furnish its proof in the next section. 
LEMMA 2. The solutions to the approximation scheme are uniformly bounded:

$$
\left\|p_{h}\right\|_{L^{\rho}\left(I ; H^{1}(\Omega)\right)}+\left\|q_{h}\right\|_{L^{2}\left(I ; H^{1}(\Omega)\right)} \leq C .
$$

In the proof, we will use the following:

PROPOSITION 1. If $b: \mathbb{R} \rightarrow \mathbb{R}$ is monotone nondecreasing, $b(0)=0$, and $\left\{a_{n}\right\}_{n=0}^{N}$ and $\left\{\omega_{n}\right\}_{n=0}^{N-1}$ are sequences of real numbers such that the $\omega_{n}$ are nonincreasing in $n$, then

$$
\text { (a) } \quad \sum_{n=1}^{N}\left(b\left(a_{n}\right)-b\left(a_{n-1}\right)\right) a_{n} \geq B\left(a_{N}\right)-B\left(a_{0}\right) \geq-B\left(a_{0}\right) \text {, }
$$

where $B(a)=\int_{0}^{a}(b(a)-b(\alpha)) d \alpha$, and

$$
\sum_{n=1}^{N} \sum_{k=1}^{n}\left(b\left(a_{k}\right)-b\left(a_{k-1}\right)\right) \omega_{n-k} a_{n} \geq-b\left(a_{0}\right) \sum_{n=1}^{N} a_{n} \omega_{n-1} .
$$

Proof: For part (a), note that by monotonicity, $B(a) \geq 0$ for any $a \in \mathbb{R}$ and

$$
\left(b\left(a_{n}\right)-b\left(a_{n-1}\right)\right) a_{n} \geq B\left(a_{n}\right)-B\left(a_{n-1}\right)
$$

hence, the sum on $n$ collapses to give the result. We turn to part (b). By induction on $N$ for the first equality,

$$
\begin{aligned}
\sum_{n=1}^{N} \sum_{k=1}^{n}\left(b\left(a_{k}\right)-b\left(a_{k-1}\right)\right) \omega_{n-k} a_{n} & =\sum_{\ell=0}^{N-1} \sum_{k=1}^{N-\ell}\left(b\left(a_{k}\right)-b\left(a_{k-1}\right)\right) a_{k+\ell} \omega_{\ell} \\
& =\sum_{\ell=0}^{N-1}\left(\mu_{\ell}-\mu_{\ell+1}-b\left(a_{0}\right) a_{\ell+1}\right) \omega_{\ell}
\end{aligned}
$$

where

$$
\mu_{\ell}=\sum_{k=1}^{N-\ell} b\left(a_{k}\right) a_{k+\ell}
$$

Note that $b$ being monotone, the $b\left(a_{k}\right)$ and $a_{k}$ are ordered similarly; consequently, $\mu_{0} \geq \mu_{\ell}$ for all $\ell$ (see [19; Theorem 368]).

Part (b) will hold once we show more generally that

$$
\sum_{\ell=0}^{N-1}\left(\mu_{\ell}-\mu_{\ell+1}\right) \omega_{\ell} \geq 0
$$


for any sequence of real numbers $\left\{\mu_{\ell}\right\}_{\ell=0}^{N}$ such that $\mu_{0} \geq \mu_{\ell}$ for all $\ell$. We proceed by induction on $N$. The result is trivial for $N=1$. Suppose the result for some $N \geq 1$, then

$$
\sum_{\ell=0}^{N}\left(\mu_{\ell}-\mu_{\ell+1}\right) \omega_{\ell}=\sum_{\ell=0}^{N-1}\left(\mu_{\ell}-\mu_{\ell+1}\right) \omega_{\ell}+\left(\mu_{N}-\mu_{N+1}\right) \omega_{N}
$$

is nonnegative if $\mu_{N} \geq \mu_{N+1}$. Suppose the contrary and replace $\omega_{N}$ by $\omega_{N-1}$; it follows that

$$
\sum_{\ell=0}^{N}\left(\mu_{\ell}-\mu_{\ell+1}\right) \omega_{\ell} \geq \sum_{\ell=0}^{N-1}\left(\mu_{\ell}-\mu_{\ell+1}\right) \omega_{\ell}+\left(\mu_{N}-\mu_{N+1}\right) \omega_{N-1}=\sum_{\ell=0}^{N-1}\left(\bar{\mu}_{\ell}-\bar{\mu}_{\ell+1}\right) \omega_{\ell} \geq 0
$$

by induction, where $\bar{\mu}_{\ell}=\mu_{\ell}$ for $\ell<N$ and $\bar{\mu}_{N}=\mu_{N+1}$.

Proof of LEMMA 2: For any integer $n$ between 1 and $N$, let $\xi=\left(p_{h}-p_{h}^{\mathrm{D}}\right) \chi_{I_{n}}(t) \epsilon$ $\ell_{h}(V)$ in (4.3) to obtain immediately

$$
\begin{aligned}
& c \int_{I_{n}}\left[\left\|\nabla p_{h}\right\|_{0}^{2}+\left(a\left(s_{h}\right) p_{h}, p_{h}\right)_{\Gamma_{\mathrm{p}}^{2}}\right] \\
& \leq C \int_{I_{n}}\left[\left\|p_{h}^{\mathrm{D}}\right\|_{1}^{2}+\left\|\gamma_{1}\right\|_{0}^{2}+\|\| f\left\|_{V^{*}}^{2}+\right\| G \|_{-\frac{1}{2}, \Gamma_{\mathrm{p}}^{2}}^{2}+\sum_{j}\left(g_{j}^{\mathrm{W}}\right)^{2}\right]+\epsilon \int_{I_{n}}\left\|p_{h}\right\|_{0}^{2}
\end{aligned}
$$

for any $\epsilon>0$, where $c$ is independent of $\epsilon$. The $L^{2}(\Omega)$-norm of $p_{h}$ is controlled by variants of Poincaré's inequality (see, e.g., [24; Theorems 3.6.4-5]):

$$
\left\|p_{h}\right\|_{0} \leq C\left\{\left\|\nabla p_{h}\right\|_{0}+\left\|p_{h}\right\|_{0, \Gamma_{\mathrm{p}}^{2, *}}+\left\|p_{h}^{\mathrm{D}}\right\|_{1}\right\}
$$

for any possible combination of the boundary conditions. Clearly

$$
\left\|p_{h}^{\mathrm{D}}\right\|_{L^{2}\left(I_{n} ; H^{1}(\Omega)\right)} \leq\left\|p^{\mathrm{D}}\right\|_{L^{2}\left(I_{n} ; H^{1}(\Omega)\right)},
$$

so we can replace $p_{h}^{\mathrm{D}}$ by $p^{\mathrm{D}}$ in (4.7). Since $p_{h}$ is piecewise constant in time, we are now able to bound it in the proper norm independently of $h$ (with Hölder's inequality if $\rho<\infty$ ).

Next, let $\zeta=q_{h}-q_{h}^{\mathrm{D}} \in \ell_{h}(W)$ in (4.4); since $\left\|q_{h}\right\|_{0, \Omega_{\boldsymbol{T}}} \leq T^{1 / 2}\left\|q^{*}\right\|_{0}$, and with the result from (4.7), immediately

$$
\begin{aligned}
c \int_{I}\left\|\nabla q_{h}\right\|_{0}^{2} \leq C\{ & \left\|q^{*}\right\|_{0}^{2}+\int_{I}\left[\left\|q_{h}^{\mathrm{D}}\right\|_{1}^{2}+\left\|\gamma_{2}\right\|_{0}^{2}+\left\|f_{\mathrm{w}}\right\|_{W^{*}}^{2}+\left\|G_{\mathrm{w}}\right\|_{-\frac{1}{2}, \Gamma_{\mathrm{s}}^{2}}^{2}\right. \\
& \left.\left.+\left\|p^{\mathrm{D}}\right\|_{1}^{2}+\left\|\gamma_{1}\right\|_{0}^{2}+\|f\|_{V^{*}}^{2}+\|G\|_{-\frac{1}{2}, \Gamma_{\mathrm{p}}^{2}}^{2}+\sum_{j}\left(g_{j}^{\mathrm{W}}\right)^{2}\right]\right\} \\
& -\int_{I}\left(\partial^{-h}\left[\phi s_{h}+\hat{\phi} \sigma\left(s_{h}\right)+\left(\sigma\left(s_{h}\right)-\sigma\left(s^{0}\right)\right) *_{h} \omega\right], q_{h}-q_{h}^{\mathrm{D}}\right) .
\end{aligned}
$$


By Proposition 1, we have that

$$
\begin{gathered}
\int_{I}\left(\partial^{-h}\left[\phi s_{h}+\hat{\phi} \sigma\left(s_{h}\right)+\left(\sigma\left(s_{h}\right)-\sigma\left(s^{0}\right)\right) *_{h} \omega\right], q_{h}\right) \\
=\sum_{n=1}^{N}\left(\phi\left(s_{h}^{n}-s_{h}^{n-1}\right)+\hat{\phi}\left(\sigma\left(s_{h}^{n}\right)-\sigma\left(s_{h}^{n-1}\right)\right), q_{h}^{n}\right) \\
\quad+\sum_{n=1}^{N} \sum_{k=1}^{n}\left(\left(\sigma\left(s_{h}^{k}\right)-\sigma\left(s_{h}^{k-1}\right)\right) \omega_{n-k}, q_{h}^{n}\right) \geq-C .
\end{gathered}
$$

Summation by parts yields

$$
\begin{aligned}
\int_{I}\left(\partial^{-h}\left[\phi s_{h}+\hat{\phi} \sigma\left(s_{h}\right)+\left(\sigma\left(s_{h}\right)-\sigma\left(s^{0}\right)\right) *_{h} \omega\right], q_{h}^{\mathrm{D}}\right) \\
=\left(\phi s_{h}^{N}+\hat{\phi} \sigma\left(s_{h}^{N}\right)+\left[\left(\sigma\left(s_{h}\right)-\sigma\left(s^{0}\right)\right) *_{h} \omega\right]^{N}, q_{h}^{\mathrm{D}, N}\right)-\left(\phi s_{h}^{0}+\hat{\phi} \sigma\left(s_{h}^{0}\right), q_{h}^{\mathrm{D}, 1}\right) \\
\quad-\int_{0}^{T-h}\left(\phi s_{h}+\hat{\phi} \sigma\left(s_{h}\right)+\left(\sigma\left(s_{h}\right)-\sigma\left(s^{0}\right)\right) *_{h} \omega, \partial^{h} q_{h}^{\mathrm{D}}\right) \\
\leq C\left\{\left\|q_{h}^{\mathrm{D}}\right\|_{L^{\infty}\left(I ; L^{1}(\Omega)\right)}+\int_{0}^{T-h}\left\|\partial^{h} q_{h}^{\mathrm{D}}\right\|_{L^{1}(\Omega)}\right\} .
\end{aligned}
$$

Now easily

$$
\begin{aligned}
\left\|q_{h}^{\mathrm{D}}\right\|_{L^{2}\left(I ; H^{1}(\Omega)\right)} & \leq\left\|q^{\mathrm{D}}\right\|_{L^{2}\left(I ; H^{1}(\Omega)\right)}, \\
\left\|q_{h}^{\mathrm{D}}\right\|_{L^{\infty}\left(I ; L^{1}(\Omega)\right)} & \leq C\left\|q^{\mathrm{D}}\right\|_{W^{1,1}\left(I ; L^{1}(\Omega)\right)},
\end{aligned}
$$

and, for any positive integer $k \leq N$ (here we need only $k=1$ ),

$$
\begin{aligned}
\int_{0}^{T-k h}\left\|\partial^{k h} q_{h}^{\mathrm{D}}\right\|_{L^{1}(\Omega)} & =\sum_{i=1}^{N-k} \frac{1}{k}\left\|\sum_{j=i}^{i+k-1}\left(q_{h}^{\mathrm{D}, j+1}-q_{h}^{\mathrm{D}, j}\right)\right\|_{L^{1}(\Omega)} \\
& =\sum_{i=1}^{N-k} \frac{1}{k h}\left\|\int_{t_{i-1}}^{t_{i+k-1}} \int_{t}^{t+h} \partial_{t} q^{\mathrm{D}}(\cdot, \tau) d \tau d t\right\|_{L^{1}(\Omega)} \\
& \leq \int_{I}\left\|\partial_{t} q^{\mathrm{D}}\right\|_{L^{1}(\Omega)}
\end{aligned}
$$

Upon combining these results, we obtain the desired bound for $q_{h}$.

Corollary 1. For any $\bar{\rho} \in[2, \rho]$ subject to $\bar{\rho}<\infty$, there exist functions $p \in$ $L^{\rho}(I ; V)+p^{\mathrm{D}}$ and $q \in L^{2}(I ; W)+q^{\mathrm{D}}$ such that, for a subsequence, $p_{h} \rightarrow p$ weakly in $L^{\bar{\rho}}\left(I ; H^{1}(\Omega)\right)$ and $q_{h} \rightarrow q$ weakly in $L^{2}\left(I ; H^{1}(\Omega)\right)$ as $h \rightarrow 0^{+}$. Moreover,

$$
0 \leq q(x, t) \leq q^{*}(x) \quad \text { a.e. on } \Omega_{T} \text {. }
$$


Proof: From Lemma $2, q_{h}-q_{h}^{\mathrm{D}}$ converges weakly in $L^{2}(I ; V)$. But $q_{h}^{\mathrm{D}} \rightarrow q^{\mathrm{D}}$ in $L^{2}\left(I ; H^{1}(\Omega)\right)$; hence, $q_{h} \rightarrow q$ in $L^{2}\left(I ; H^{1}(\Omega)\right)$, where $q \in L^{2}(I ; V)+q^{\mathrm{D}}$. If $\rho<\infty$, similar reasoning obtains $p$. If $\rho=\infty$, we obtain weak convergence of $p_{h}$ in $L^{\bar{\rho}}\left(I ; H^{1}(\Omega)\right)$ for any $\bar{\rho}<\infty$. Also $\|p\|_{L^{\bar{p}}\left(I ; H^{1}(\Omega)\right)} \leq C$ with $C$ independent of $\bar{\rho}$, so in fact $p \in L^{\infty}\left(I ; H^{1}(\Omega)\right)$. Trivially (4.11) follows from (4.5).

LEmma 3. Independently of $h$, as $\eta \rightarrow 0^{+}$,

$$
\int_{\eta}^{T}\left(\phi\left[s_{h}(\cdot, t)-s_{h}(\cdot, t-\eta)\right], q_{h}(\cdot, t)-q_{h}(\cdot, t-\eta)\right) d t=o(\eta) .
$$

Proof: Let $k$ be an integer between 1 and $N$, and fix $\tau \in(k h, T]$. Define $j$ by $\tau \in I_{j}$ and define the interval $R=R(\tau)=((j-k) h, j h]$. Take

$$
\zeta(x, t)=k h \chi_{R}(t) \partial^{-k h}\left(q_{h}-q_{h}^{\mathrm{D}}\right)(x, \tau) \in \ell_{h}(W)
$$

in (4.4). Note that

$$
\begin{aligned}
& \int_{I} \partial^{-h}\left(\phi s_{h}+\hat{\phi} \sigma\left(s_{h}\right)\right) \chi_{R(\tau)} \\
& \quad=\phi\left[s_{h}(\cdot, \tau)-s_{h}(\cdot, \tau-k h)\right]+\hat{\phi}\left[\sigma\left(s_{h}(\cdot, \tau)\right)-\sigma\left(s_{h}(\cdot, \tau-k h)\right)\right] .
\end{aligned}
$$

Then, with Lemma 2, the fact that the measure of $R$ is $k h$, and (4.10), we obtain upon integrating in $\tau$

$$
\begin{gathered}
\int_{k h}^{T}\left(\phi\left[s_{h}(\cdot, \tau)-s_{h}(\cdot, \tau-k h)\right], q_{h}(\cdot, \tau)-q_{h}(\cdot, \tau-k h)\right) d \tau \\
\leq C k h-\int_{k h}^{T} \int_{R(\tau)}\left(\partial^{-h}\left[\left(\sigma\left(s_{h}\right)-\sigma\left(s^{0}\right)\right) * h \omega\right](\cdot, t)\right. \\
\left.q_{h}(\cdot, \tau)-q_{h}(\cdot, \tau-k h)\right) d t d \tau
\end{gathered}
$$

Now by induction on $k$, we can verify easily that

$$
\begin{aligned}
\int_{R(\tau)} & \partial^{-h}\left[\left(\sigma\left(s_{h}\right)-\sigma\left(s^{0}\right)\right) *_{h} \omega\right](\cdot, t) d t \\
= & \sum_{i=j-k+1}^{j} \sum_{m=1}^{i}\left(\sigma\left(s_{h}^{m}\right)-\sigma\left(s_{h}^{m-1}\right)\right) \omega_{i-m} \\
= & \sum_{\ell=1}^{j}\left(\sigma\left(s_{h}^{\ell}\right)-\sigma\left(s_{h}^{\ell-k}\right)\right) \omega_{j-\ell},
\end{aligned}
$$


since $s_{h}^{m}=s^{0}$ for $m \leq 0$. So

$$
\begin{aligned}
& -\int_{k h}^{T} \int_{R(\tau)} \partial^{-h}\left[\left(\sigma\left(s_{h}\right)-\sigma\left(s^{0}\right)\right) *_{h} \omega\right](\cdot, t)\left(q_{h}(\cdot, \tau)-q_{h}(\cdot, \tau-k h)\right) d t d \tau \\
& =-\sum_{j=k+1}^{N} \sum_{\ell=1}^{j}\left(\sigma\left(s_{h}^{\ell}\right)-\sigma\left(s_{h}^{\ell-k}\right)\right) \omega_{j-\ell}\left(q_{h}^{j}-q_{h}^{j-k}\right) h \\
& =-\sum_{j=k+1}^{N}\left\{\sum_{\ell=1}^{j-k} \sigma\left(s_{h}^{\ell}\right)\left(\omega_{j-\ell}-\omega_{j-\ell-k}\right)\right. \\
& \left.\quad+\sum_{\ell=j-k+1}^{j} \sigma\left(s_{h}^{\ell}\right) \omega_{j-\ell}-\sum_{\ell=1}^{k} \sigma\left(s^{0}\right) \omega_{j-\ell}\right\}\left(q_{h}^{j}-q_{h}^{j-k}\right) h \\
& \leq 2 q^{*} \sum_{j=k+1}^{N}\left\{\sum_{\ell=1}^{j-k}\left(\omega_{j-\ell-k}-\omega_{j-\ell}\right)+\sum_{\ell=j-k+1}^{j} \omega_{j-\ell}+\sum_{\ell=1}^{k} \omega_{j-\ell}\right\} h \\
& =4 q^{*} \sum_{j=k+1}^{N} \sum_{m=0}^{k-1} \omega_{m} h \leq 4 T q^{*} \int_{0}^{k h} \omega(\cdot, \tau) d \tau .
\end{aligned}
$$

Since $\omega \in L^{1}\left(\Omega_{T}\right)$, independently of $h$,

$$
\int_{0}^{\eta}\|\omega(\cdot, \tau)\|_{L^{1}(\Omega)} d \tau=o(\eta)
$$

and so (4.13) combined with (4.15) gives the lemma for $\eta=k h$. Arbitrary $\eta$ are now allowed because we are dealing with step functions in time.

REMARK: If $\omega \equiv 0$, then we can replace $o(\eta)$ by $C \eta$ in (4.12). In that case, if $\mathcal{S}^{-1}(s)$ were Hölder continuous of order $(r-1)^{-1}$ in $s(2 \leq r<\infty)$, we would conclude that

$$
\int_{\eta}^{T}\left\|q_{h}(\cdot, t)-q_{h}(\cdot, t-\eta)\right\|_{L^{r}(\Omega)}^{r} d t \leq C \eta,
$$

and hence, for $0<\epsilon<1 / r$ and uniformly in $h, q_{h} \in W^{1 / r-\epsilon, r}\left(I ; L^{r}(\Omega)\right) \cap$ $L^{2}\left(I ; H^{1}(\Omega)\right)$, which would further imply that $q_{h} \rightarrow q$ strongly in $L^{2}\left(\Omega_{T}\right)$. This conclusion can be reached without additional assumption on $\mathcal{S}^{-1}$. However, this calculation suggests that $q$ (and hence $s$ ) cannot be expected to have a trace on $\Omega \times\{0\}$, so (3.6) is a proper way to apply the initial condition.

LEMma 4. For a subsequence, $q_{h} \rightarrow q$ strongly in $L^{2}\left(\Omega_{T}\right)$ as $h \rightarrow 0^{+}$.

An auxiliary result given in Section 6 below, namely Theorem 3, is used in the proof, so we present it there. 
Corollary 2. For fixed $\epsilon \in\left(0, \frac{1}{2}\right)$, for a subsequence, $q_{h} \rightarrow q$ strongly both in $L^{2}\left(I ; H^{1-\epsilon}(\Omega)\right)$ and in $L^{2}\left(I ; H^{1 / 2-\epsilon}(\partial \Omega)\right)$. Moreover, with $s=\mathcal{S}(q), s_{h} \rightarrow s$ pointwise a.e. on either $\Omega_{T}$ or $\partial \Omega \times I$.

Proof: By a well-known interpolation inequality, namely,

$$
\|u\|_{r} \leq \epsilon\|u\|_{1}+C_{\epsilon}\|u\|_{0}
$$

for any $0<r<1$ and $\epsilon>0$, and by the boundedness of the trace operator, $q_{h} \rightarrow q$ as asserted. The pointwise convergence follows since $\mathcal{S}(x, q)$ is continuous in $q$.

We have already obtained (3.2)-(3.3). We can now obtain convergence of (4.3)(4.4) for a subsequence. Since $\bigcup_{N=1}^{\infty} \ell_{h}(V)$ is dense in $L^{\rho^{*}}(I ; V)$, we obtain (3.4) as well as

$$
\begin{aligned}
\lim _{h \rightarrow 0^{+}} \int_{I}\left(\partial^{-h}\left[\phi s_{h}+\hat{\phi} \sigma\left(s_{h}\right)+\left(\sigma\left(s_{h}\right)-\sigma\left(s^{0}\right)\right) *_{h} \omega\right], \zeta\right) \\
\quad+\int_{I}\left(\mathbf{k}\left[\lambda(s) \nabla q+\lambda_{\mathbf{w}}(s) \nabla p+\gamma_{2}(s)\right], \nabla \zeta\right) \\
\quad+\int_{I}\left(G_{\mathbf{w}}(s)+a_{\mathbf{w}}(s) p, \zeta\right)_{\Gamma_{\mathbf{s}}^{2}} \\
=\int_{I}\left(f_{\mathbf{w}}(s), \zeta\right) \quad \text { for all } \zeta \in \bigcup_{N=1}^{\infty} \ell_{h}(W) .
\end{aligned}
$$

For any $\zeta \in L^{2}(I ; W), \zeta_{h} \in \ell_{h}(W)$ where $\zeta_{h}(x, t)=h^{-1} \int_{I_{k}} \zeta(x, \tau) d \tau$ for $t \in I_{k}$. Hence from (4.4),

$$
\int_{I}\left(\partial^{-h}\left[\phi s_{h}+\hat{\phi} \sigma\left(s_{h}\right)+\left(\sigma\left(s_{h}\right)-\sigma\left(s^{0}\right)\right) *_{h} \omega\right], \zeta\right) \leq C\left\|\zeta_{h}\right\|_{L^{2}(I ; W)} \leq C\|\zeta\|_{L^{2}(I ; W)} ;
$$

that is, a subsequence of $\partial^{-h}\left[\phi s_{h}+\hat{\phi} \sigma\left(s_{h}\right)+\left(\sigma\left(s_{h}\right)-\sigma\left(s^{0}\right)\right) *_{h} \omega\right]$ converges weakly in $L^{2}(I ; W)$. But if now $\zeta \in C_{0}^{\infty}\left(\Omega_{T}\right)$, then for sufficiently small $h$,

$$
\begin{aligned}
\int_{I}\left(\partial^{-h}\right. & {\left.\left[\phi s_{h}+\hat{\phi} \sigma\left(s_{h}\right)+\left(\sigma\left(s_{h}\right)-\sigma\left(s^{0}\right)\right) *_{h} \omega\right], \zeta\right) } \\
= & -\int_{0}^{T-h}\left(\phi s_{h}+\hat{\phi} \sigma\left(s_{h}\right)+\left(\sigma\left(s_{h}\right)-\sigma\left(s^{0}\right)\right) *_{h} \omega, \partial^{h} \zeta\right) \\
\longrightarrow & -\int_{I}\left(\phi s+\hat{\phi} \sigma(s)+\left(\sigma(s)-\sigma\left(s^{0}\right)\right) * \omega, \partial_{t} \zeta\right) \\
& =\int_{I}\left\langle\partial_{t}\left[\phi s+\hat{\phi} \sigma(s)+\left(\sigma(s)-\sigma\left(s^{0}\right)\right) * \omega\right], \zeta\right\rangle,
\end{aligned}
$$


where we have used the fact that

$$
\left(\sigma\left(s_{h}\right)-\sigma\left(s^{0}\right)\right) *_{h} \omega \longrightarrow\left(\sigma(s)-\sigma\left(s^{0}\right)\right) * \omega
$$

in the $L^{\infty}\left(\Omega_{T}\right)$ weak-* topology. Thus (3.5) follows from (4.17).

Finally, if $\zeta \in L^{2}(I ; W) \cap W^{1,1}\left(I ; L^{1}(\Omega)\right)$ satisfies $\zeta(x, T)=0$, then

$$
\begin{gathered}
\int_{I}\left(\partial^{-h}\left[\phi s_{h}+\hat{\phi} \sigma\left(s_{h}\right)+\left(\sigma\left(s_{h}\right)-\sigma\left(s^{0}\right)\right) *_{h} \omega\right], \zeta\right) \\
\quad+\int_{0}^{T-h}\left(\phi\left(s_{h}-s^{0}\right)+\hat{\phi}\left(\sigma\left(s_{h}\right)-\sigma\left(s^{0}\right)\right)+\left(\sigma\left(s_{h}\right)-\sigma\left(s^{0}\right)\right) *_{h} \omega, \partial^{h} \zeta\right) \\
=\frac{1}{h} \int_{T-h}^{T}\left(\phi\left(s_{h}-s^{0}\right)+\hat{\phi}\left(\sigma\left(s_{h}\right)-\sigma\left(s^{0}\right)\right)+\left(\sigma\left(s_{h}\right)-\sigma\left(s^{0}\right)\right) *_{h} \omega, \zeta\right) .
\end{gathered}
$$

This converges to give (3.6), and the proof of Theorem 1 is complete.

\section{Proof of Lemma 1}

For technical reasons, in this section we must allow for the possibility that $s \notin$ $[0,1]$. All functions of $s$ are extended constantly outside $[0,1]$ except $\mathcal{S}$, which is extended outside this interval such that $\partial \mathcal{S} / \partial q=1$; hence, in (5.2) below we mean that at $x \in \Omega$,

$$
s=\text { extended } \mathcal{S}(q)=\left\{\begin{array}{llr}
q & \text { for } & q<0 \\
\mathcal{S}(x, q) & \text { for } & 0 \leq q \leq q^{*}(x) \\
q-q^{*}(x)+1 & \text { for } & q^{*}(x)<q .
\end{array}\right.
$$

Obviously, $q$ and $s$ follow each other up to a constant:

Proposition 2. If $x \in \Omega$ and $q \in \mathbb{R}$, then for $s=\mathcal{S}(x, q)$,

$$
|s| \leq 1+|q| \quad \text { and } \quad|q| \leq q^{*}(x)+|s| \text {. }
$$

We show the existence of a solution to the finite difference scheme by induction on the time levels. At each time, we have an elliptic problem. The main difficulty in proving Lemma 1 lies in treating the matrix source operator. To treat this delay operator, let $n$ be a fixed positive integer and let $\left\{\tilde{\sigma}^{1}(x), \ldots, \tilde{\sigma}^{n-1}(x), \tilde{\sigma}^{n}(s)\right\}$ be a sequence of functions such that $\tilde{\sigma}^{k}: \Omega \rightarrow[0,1]$ for $k<n$ is assumed known and $\tilde{\sigma}^{n}(s)=\sigma(s)$. Then the time discrete matrix source operator at time $t_{n}$ is

$$
\hat{\phi} \tilde{\sigma}^{n}+\sum_{k=1}^{n}\left(\tilde{\sigma}^{k}-\tilde{\sigma}^{0}\right) \omega_{n-k},
$$


and its backward difference is equal to

$$
\begin{aligned}
\frac{1}{h}\left\{\hat{\phi}\left(\tilde{\sigma}^{n}-\tilde{\sigma}^{n-1}\right)+\sum_{k=1}^{n}\left(\tilde{\sigma}^{k}-\tilde{\sigma}^{0}\right) \omega_{n-k}-\sum_{k=1}^{n-1}\left(\tilde{\sigma}^{k}-\tilde{\sigma}^{0}\right) \omega_{n-k-1}\right\} \\
=\frac{1}{h}\left\{\hat{\phi}\left(\sigma(s)-\tilde{\sigma}^{n-1}\right)+\sigma(s) \omega_{0}-\tilde{\sigma}^{0} \omega_{n-1}+\sum_{k=1}^{n-1} \tilde{\sigma}^{k}\left(\omega_{n-k}-\omega_{n-k-1}\right)\right\} .
\end{aligned}
$$

Lemma 1 is a corollary of the following theorem.

THEOREM 2. Assume (A1)-(A12) with all functions independent of time. Let $n$ and $\left\{\tilde{\sigma}^{k}\right\}_{k=0}^{n}$ be as above, let $\tilde{s}: \Omega \rightarrow[0,1]$, and suppose that $\left\{\omega_{k}(x)\right\}_{k=0}^{n-1}$ is a nonnegative, nonincreasing sequence for each $x \in \Omega$. Then for $h$ sufficiently small, the weak problem

$$
\begin{aligned}
& s=\mathcal{S}(q), \\
& \left(\mathbf{k}\left[\lambda(s) \nabla p+\gamma_{1}(s)\right], \nabla \xi\right)+(G(s)+a(s) p, \xi)_{\Gamma_{\mathrm{p}}^{2}}+\left.\sum_{j} g_{j}^{\mathrm{W}} \xi\right|_{\Gamma_{\mathrm{p}}^{3, j}} \\
& =(f(s), \xi) \quad \text { for all } \xi \in V, \\
& \frac{1}{h}\left(\phi(s-\tilde{s})+\hat{\phi}\left(\sigma(s)-\tilde{\sigma}^{n-1}\right)\right. \\
& \left.+\sigma(s) \omega_{0}-\tilde{\sigma}^{0} \omega_{n-1}+\sum_{k=1}^{n-1} \tilde{\sigma}^{k}\left(\omega_{n-k}-\omega_{n-k-1}\right), \zeta\right) \\
& +\left(\mathbf{k}\left[\lambda(s) \nabla q+\lambda_{\mathbf{w}}(s) \nabla p+\gamma_{2}(s)\right], \nabla \zeta\right)+\left(G_{\mathbf{w}}(s)+a_{\mathbf{w}}(s) p, \zeta\right)_{\Gamma_{\mathbf{s}}^{2}} \\
& =\left(f_{\mathrm{w}}(s), \zeta\right) \quad \text { for all } \zeta \in W
\end{aligned}
$$

has a solution $p \in V+p^{\mathrm{D}}$ and $q \in W+q^{\mathrm{D}}$ such that $0 \leq q(x) \leq q^{*}(x)$ a.e. on $\Omega$.

ProOF: Let $\left\{v_{m}\right\}_{m=1}^{\infty}$ and $\left\{w_{m}\right\}_{m=1}^{\infty}$ form bases for $V$ and $W$, respectively. Set $V_{m}=\bigcup_{k=1}^{m}\left\{v_{k}\right\}$ and $W_{m}=\bigcup_{k=1}^{m}\left\{w_{k}\right\}$. We define the Galerkin procedure corresponding to (5.2)-(5.4) by subscripting $p, q, s, V$, and $W$ by $m$.

Define $\Phi_{m}: \mathbb{R}^{2 m} \rightarrow \mathbb{R}^{2 m}$ by $\Phi_{m}\left(\begin{array}{c}\alpha \\ \beta\end{array}\right)=\left(\begin{array}{c}\bar{\alpha} \\ \bar{\beta}\end{array}\right)$ where, with $v=\sum_{k=1}^{m} \alpha_{k} v_{k}$, 


$$
\begin{aligned}
w=\sum_{k=1}^{m} \beta_{k} w_{k}, \text { and } \varsigma=\mathcal{S}\left(w+q^{\mathrm{D}}\right), \\
\bar{\alpha}_{i}=\left(\mathbf{k}\left[\lambda(\varsigma) \nabla\left(v+p^{\mathrm{D}}\right)+\gamma_{1}(\varsigma)\right], \nabla v_{i}\right) \\
\quad+\left(G(\varsigma)+a(\varsigma)\left(v+p^{\mathrm{D}}\right), v_{i}\right)_{\Gamma_{\mathbf{p}}^{2}}+\left.\sum_{j} g_{j}^{\mathrm{W}} v_{i}\right|_{\Gamma_{\mathbf{p}}^{3, j}}-\left(f(\varsigma), v_{i}\right), \\
\bar{\beta}_{j}=\frac{1}{h}\left(\phi(\varsigma-\tilde{s})+\hat{\phi}\left(\sigma(\varsigma)-\tilde{\sigma}^{n-1}\right)\right. \\
\left.\quad+\sigma(\varsigma) \omega_{0}-\tilde{\sigma}^{0} \omega_{n-1}+\sum_{k=1}^{n-1} \tilde{\sigma}^{k}\left(\omega_{n-k}-\omega_{n-k-1}\right), w_{j}\right) \\
+\left(\mathbf{k}\left[\lambda(\varsigma) \nabla\left(w+q^{\mathrm{D}}\right)+\lambda_{\mathbf{w}}(\varsigma) \nabla\left(v+p^{\mathrm{D}}\right)+\gamma_{2}(\varsigma)\right], \nabla w_{j}\right) \\
+\left(G_{\mathbf{w}}(\varsigma)+a_{\mathbf{w}}(\varsigma)\left(v+p^{\mathrm{D}}\right), w_{j}\right)_{\Gamma_{\mathbf{s}}^{2}}-\left(f_{\mathbf{w}}(\varsigma), w_{j}\right) .
\end{aligned}
$$

By assumption, $\Phi_{m}$ is continuous. We obtain easily for any fixed $\epsilon>0$,

$$
\begin{aligned}
\Phi_{m}\left(\begin{array}{c}
\alpha \\
\beta
\end{array}\right) \cdot\left(\begin{array}{c}
\alpha \\
\beta
\end{array}\right) \geq & c\left\{\|\nabla v\|_{0}^{2}+\|\nabla w\|_{0}^{2}+\|v\|_{0, \Gamma_{\mathrm{p}}^{2, *}}^{2}\right\}+\left(a_{\mathrm{w}}(\varsigma) v, w\right)_{\Gamma_{s}^{2}} \\
- & \epsilon\left\{\|v\|_{0}^{2}+\|w\|_{0}^{2}\right\}-C\left\{\left\|p^{\mathrm{D}}\right\|_{1}^{2}+\left\|q^{\mathrm{D}}\right\|_{1}^{2}+\left\|\gamma_{1}\right\|_{0}^{2}+\left\|\gamma_{2}\right\|_{0}^{2}\right. \\
& \left.+\|f\|_{V^{*}}^{2}+\left\|f_{\mathrm{w}}\right\|_{W^{*}}^{2}+\|G\|_{-\frac{1}{2}, \Gamma_{\mathrm{p}}^{2}}^{2}+\left\|G_{\mathrm{w}}\right\|_{-\frac{1}{2}, \Gamma_{\mathrm{s}}^{2}}^{2}+\sum_{j}\left(g_{j}^{\mathrm{W}}\right)^{2}\right\} \\
+ & \frac{1}{h}\left(\phi(\varsigma-\tilde{s})+\hat{\phi}\left(\sigma(\varsigma)-\tilde{\sigma}^{n-1}\right)\right. \\
& \left.+\sigma(\varsigma) \omega_{0}-\tilde{\sigma}^{0} \omega_{n-1}+\sum_{k=1}^{n-1} \tilde{\sigma}^{k}\left(\omega_{n-k}-\omega_{n-k-1}\right), w\right) .
\end{aligned}
$$

where $c$ is independent of $\epsilon$. Proposition 2 shows that

$$
h^{-1}(\phi(\varsigma-\tilde{s}), w) \geq \frac{\phi_{*}}{2 h}\|w\|_{0}^{2}-C(h)\left\{1+\left\|q^{*}\right\|_{0}^{2}+\left\|q^{\mathrm{D}}\right\|_{0}^{2}\right\},
$$

and easily

$$
\begin{aligned}
\frac{1}{h}\left(\hat{\phi}\left(\sigma(\varsigma)-\tilde{\sigma}^{n-1}\right)+\right. & \left.\sigma(\varsigma) \omega_{0}-\tilde{\sigma}^{0} \omega_{n-1}+\sum_{k=1}^{n-1} \tilde{\sigma}^{k}\left(\omega_{n-k}-\omega_{n-k-1}\right), w\right) \\
& \geq-\frac{2}{h}\left\{\|\hat{\phi}\|_{L^{\infty}(\Omega)}+\sum_{k=0}^{n-1}\left\|\omega_{k}\right\|_{0}\right\}\|w\|_{0} \geq-\epsilon\|w\|_{0}^{2}-C(h) .
\end{aligned}
$$


With (4.16)

$$
\left|\left(a_{\mathrm{w}}(\varsigma) v, w\right)_{\Gamma_{s}^{2}}\right| \leq C\|v\|_{1}\|w\|_{0, \Gamma_{s}^{2}} \leq \epsilon\left\{\|v\|_{1}^{2}+\|w\|_{1}^{2}\right\}+C^{\prime}\|w\|_{0}^{2}
$$

for some $C^{\prime}$ independent of $h$. So with Poincaré's inequality (see (4.8) above) and with $h$ sufficiently small,

$$
\Phi_{m}\left(\begin{array}{c}
\alpha \\
\beta
\end{array}\right) \cdot\left(\begin{array}{c}
\alpha \\
\beta
\end{array}\right) \geq c\left\{\|v\|_{1}^{2}+\|w\|_{1}^{2}\right\}-C(h) .
$$

This is strictly positive for $|\alpha|+|\beta|$ large enough. As a consequence $\Phi_{m}$ has a zero (for otherwise we could construct a continuous deformation of the ball to the sphere); that is, there exists a solution to the Galerkin procedure.

None of the constants appearing above depend on $m$, so we have also shown that the solutions $p_{m}, q_{m}$ are uniformly bounded in $H^{1}(\Omega)$ and that (with Proposition 2) $s_{m}$ is uniformly bounded in $L^{2}(\Omega)$. We can now find $p \in V+p^{\mathrm{D}}, q \in W+q^{\mathrm{D}}$, and $s \in L^{2}(\Omega)$ such that $p_{m} \rightarrow p$ and $q_{m} \rightarrow q$ weakly in $H^{1}(\Omega)$ and $s_{m} \rightarrow s$ weakly in $L^{2}(\Omega)$ (for a subsequence) as $m \rightarrow \infty$. Furthermore, $q_{m} \rightarrow q$ strongly in $H^{1-\epsilon}(\Omega)$, so $s_{m}=\mathcal{S}\left(q_{m}\right) \rightarrow \mathcal{S}(q)=s$ pointwise a.e. on $\Omega$ and on $\partial \Omega$. Thus, $p, q$ is a solution of $(5.2)-(5.4)$.

To establish the maximum principle, first take $\zeta=q^{-}=\min (q, 0) \in W$ in (5.4) to obtain that

$$
\begin{aligned}
h^{-1}\left(\phi(s-\tilde{s})+\hat{\phi}\left(\sigma(s)-\tilde{\sigma}^{n-1}\right), q^{-}\right) \\
\quad+\frac{1}{h}\left(\sigma(s) \omega_{0}-\tilde{\sigma}^{0} \omega_{n-1}+\sum_{k=1}^{n-1} \tilde{\sigma}^{k}\left(\omega_{n-k}-\omega_{n-k-1}\right), q^{-}\right) \\
=-\left(\mathbf{k}\left[\lambda(s) \nabla q+\lambda_{\mathbf{w}}(s) \nabla p+\gamma_{2}(s)\right], \nabla q^{-}\right) \\
\quad-\left(G_{\mathbf{w}}(s)+a_{\mathbf{w}}(s) p, q^{-}\right)_{\Gamma_{\mathbf{s}}^{2}}+\left(f_{\mathbf{w}}(s), q^{-}\right) \\
\leq 0
\end{aligned}
$$

using (A4) and (A7). The second term on the far left side of (5.5) is nonnegative because $\sigma(s) q^{-}=0$ and the $\omega_{k}$ decrease in $k$. The first term is also nonnegative, and so we conclude that $q \geq 0$.

Next take $\zeta=\left(q-q^{*}\right)^{+}=\max \left(q-q^{*}, 0\right) \in W$ in (5.4) and subtract (5.3) with $\xi=\zeta$ (or $\xi=\zeta-\int_{\Omega} \zeta d x$ if $\Gamma_{\mathrm{p}}^{1}=\emptyset$ and $\left.a \equiv 0\right) ; \xi \in V$ by (A1). We obtain (with (A8)) that

$$
\begin{aligned}
h^{-1}\left(\phi(s-\tilde{s})+\hat{\phi}\left(\sigma(s)-\tilde{\sigma}^{n-1}\right),\left(q-q^{*}\right)^{+}\right) \\
\quad+\frac{1}{h}\left(\sigma(s) \omega_{0}-\tilde{\sigma}^{0} \omega_{n-1}+\sum_{k=1}^{n-1} \tilde{\sigma}^{k}\left(\omega_{n-k}-\omega_{n-k-1}\right),\left(q-q^{*}\right)^{+}\right) \\
=-\left(\mathbf{k}\left[\lambda(s) \nabla q-\lambda_{\mathrm{o}}(s) \nabla p+\gamma_{2}(s)-\gamma_{1}(s)\right], \nabla\left(q-q^{*}\right)^{+}\right) \\
\quad-\left(G_{\mathbf{w}}(s)-G(s)+\left(a_{\mathbf{w}}(s)-a(s)\right) p,\left(q-q^{*}\right)^{+}\right)_{\Gamma_{\mathrm{s}}^{2}}+\left(f_{\mathbf{w}}(s)-f,\left(q-q^{*}\right)^{+}\right)
\end{aligned}
$$

$\leq 0$. 
When $q \geq q^{*}, \sigma(s)=1$, so the second term on the far left side above is

$$
\begin{array}{r}
\frac{1}{h}\left(\omega_{0}-\tilde{\sigma}^{0} \omega_{n-1}+\sum_{k=1}^{n-1} \tilde{\sigma}^{k}\left(\omega_{n-k}-\omega_{n-k-1}\right),\left(q-q^{*}\right)^{+}\right) \\
\geq h^{-1}\left(\omega_{0}-\omega_{n-1}+\left(\omega_{n-1}-\omega_{0}\right),\left(q-q^{*}\right)^{+}\right)=0
\end{array}
$$

Since $s=q-q^{*}+1$ when $q \geq q^{*}$, the first term on the far left side of (5.6) is nonnegative; thus, $q \leq q^{*}$.

\section{An Auxiliary Result and Proof of Lemma 4}

In this section we consider results that are related to Lemmas 1.8 and 1.9 of Alt and Luckhaus [2].

Technical Lemma. Assuming (A1) and (A5), there exists for each $M>0$ a continuous $\mu_{M}: \mathbb{R} \rightarrow \mathbb{R}$ with $\mu_{M}(0)=0$ such that if $u_{i}$ satisfies $\left\|u_{i}\right\|_{1} \leq M$ and $0 \leq u_{i}(x) \leq q^{*}(x)$ a.e. on $\Omega$ for $i=1,2$, then

$$
\left\|u_{1}-u_{2}\right\|_{0} \leq \mu_{M}(\delta)
$$

whenever

$$
\left(\mathcal{S}\left(u_{1}\right)-\mathcal{S}\left(u_{2}\right), u_{1}-u_{2}\right) \leq \delta .
$$

Proof: Suppose the contrary result. Then there exist $M>0$ and functions $u_{i}^{\delta}$ for $\delta \rightarrow 0^{+}$satisfying the hypotheses, (6.2), and

$$
\left\|u_{1}^{\delta}-u_{2}^{\delta}\right\|_{0} \geq c>0
$$

Now, for a subsequence, $u_{i}^{\delta} \rightarrow u_{i}$ weakly in $H^{1}(\Omega)$ and strongly in $L^{2}(\Omega)$, so (6.3) holds for $u_{1}-u_{2}$. But also

$$
0^{+} \longleftarrow \delta \geq\left(\mathcal{S}\left(u_{1}^{\delta}\right)-\mathcal{S}\left(u_{2}^{\delta}\right), u_{1}^{\delta}-u_{2}^{\delta}\right) \longrightarrow\left(\mathcal{S}\left(u_{1}\right)-\mathcal{S}\left(u_{2}\right), u_{1}-u_{2}\right) \geq 0
$$

which implies that $u_{1}-u_{2}=0$, a contradiction.

For the next result, let $m$ be a positive integer, $\delta=T / m, I_{k}^{\delta}=((k-1) \delta, k \delta]$, and define the operator $A^{\delta}: L^{1}(I) \rightarrow L^{1}(I)$ by

$$
A^{\delta}(u)=\frac{1}{\delta} \int_{I_{k}^{\delta}} u(\tau) d \tau \quad \text { for } t \in I_{k}^{\delta}
$$

Theorem 3. Assume (A1), (A2), and (A5). If for all $h=T / N$ there are $u_{h} \in$ $\ell_{h}\left(H^{1}(\Omega)\right)$ satisfying

$$
\left\|u_{h}\right\|_{L^{2}\left(I ; H^{1}(\Omega)\right)} \leq C^{\prime}, \quad 0 \leq u_{h}(x, t) \leq q^{*}(x) \quad \text { a.e. on } \Omega_{T}
$$


and

$$
\int_{\eta}^{T}\left(\phi \left[\mathcal { S } \left(u_{h}(\cdot, t)-\mathcal{S}\left(u_{h}(\cdot, t-\eta)\right], u_{h}(\cdot, t)-u_{h}(\cdot, t-\eta) \leq \mu(\eta)\right.\right.\right.
$$

for some $C^{\prime}$ independent of $h$, some continuous, increasing $\mu:[0, T] \rightarrow[0, \infty)$ such that $\mu(0)=0$, and any $\eta>0$, then $A^{\delta}\left(u_{h}\right) \rightarrow u_{h}$ strongly in $L^{2}\left(\Omega_{T}\right)$ uniformly in $h$ as $\delta \rightarrow 0^{+}$.

Proof: For positive $\eta$ and $M$, define

$$
\begin{aligned}
R= & R\left(u_{h}, \eta, M\right) \\
= & \left\{t \in(\eta, T]:\left\|u_{h}(\cdot, t)\right\|_{1}+\left\|u_{h}(\cdot, t-\eta)\right\|_{1}\right. \\
& \left.\quad+\frac{1}{\mu(\eta)}\left(\phi\left[\mathcal{S}\left(u_{h}(\cdot, t)\right)-\mathcal{S}\left(u_{h}(\cdot, t-\eta)\right)\right], u_{h}(\cdot, t)-u_{h}(\cdot, t-\eta)\right)>M\right\} .
\end{aligned}
$$

Clearly the measure of $R \leq C^{\prime 2} / M^{2}$. If $t \in I \backslash R$, then by the Technical Lemma,

$$
\left\|u_{h}(\cdot, t)-u_{h}(\cdot, t-\eta)\right\|_{0} \leq \mu_{M}\left(\frac{M \mu(\eta)}{\phi_{*}}\right)
$$

consequently,

$$
\int_{\eta}^{T}\left\|u_{h}(\cdot, t)-u_{h}(\cdot, t-\eta)\right\|_{0}^{2} \leq T \mu_{M}^{2}\left(\frac{M \mu(\eta)}{\phi_{*}}\right)+\frac{4 C^{2}}{M^{2}}\left\|q^{*}\right\|_{0}^{2} .
$$

With $M$ large and $\eta$ small, we conclude that

$$
\int_{\eta}^{T}\left\|u_{h}(\cdot, t)-u_{h}(\cdot, t-\eta)\right\|_{0}^{2} \longrightarrow 0
$$

uniformly in $h$ as $\eta \rightarrow 0^{+}$.

We now estimate

$$
\begin{aligned}
\int_{I}\left\|u_{h}-A^{\delta}\left(u_{h}\right)\right\|_{0}^{2} & =\sum_{k=1}^{m} \int_{I_{k}^{\delta}}\left\|\frac{1}{\delta} \int_{I_{k}^{\delta}}\left(u_{h}(\cdot, t)-u_{h}(\cdot, \tau)\right) d \tau\right\|_{0}^{2} d t \\
& \leq \sum_{k=1}^{m} \int_{I_{k}^{\delta}} \frac{1}{\delta} \int_{t-k \delta}^{t-(k-1) \delta}\left\|u_{h}(\cdot, t)-u_{h}(\cdot, t-\eta)\right\|_{0}^{2} d \eta d t \\
& =\sum_{k=1}^{m} \frac{1}{\delta} \int_{-\delta}^{\delta} \int_{\max ((k-1) \delta, \eta+(k-1) \delta)}^{\min (k \delta+\eta, k \delta)}\left\|u_{h}(\cdot, t)-u_{h}(\cdot, t-\eta)\right\|_{0}^{2} d t d \eta \\
& \leq \frac{2}{\delta} \int_{0}^{\delta} \int_{\eta}^{T}\left\|u_{h}(\cdot, t)-u_{h}(\cdot, t-\eta)\right\|_{0}^{2} d t d \eta,
\end{aligned}
$$


which tends to zero uniformly in $h$ as $\delta \rightarrow 0^{+}$by (6.4).

Proof of Lemma 4: Clearly $\left\|A^{\delta}\left(q_{h}\right)\right\|_{L^{2}\left(I ; H^{1}(\Omega)\right)} \leq C$ for all $h$, so for fixed $\delta>0$, $A^{\delta}\left(q_{h}\right)$ converges strongly in $L^{2}\left(\Omega_{T}\right)$ as $h \rightarrow 0^{+}$. Therefore

$$
\left\|q_{h_{1}}-q_{h_{2}}\right\|_{0, \Omega_{T}} \leq \sum_{i=1}^{2}\left\|q_{h_{i}}-A^{\delta}\left(q_{h_{i}}\right)\right\|_{0, \Omega_{T}}+\left\|A^{\delta}\left(q_{h_{1}}\right)-A^{\delta}\left(q_{h_{2}}\right)\right\|_{0, \Omega_{T}}
$$

shows the lemma by Theorem 3 .

\section{A Remark on the Solution When $F(s)=\hat{\phi} \partial_{t} \sigma(s)$}

When $F(s)=\hat{\phi} \partial_{t} \sigma(s)$ is not a delay operator, the existence of a solution follows directly from the results for an unfractured reservoir $(F(s) \equiv 0)$. To see this, define the pseudo-saturation variable [17] as

$$
S(x, t)=\phi_{\mathrm{t}}^{-1}[\phi s+\hat{\phi} \sigma(s)]
$$

where $\phi_{\mathrm{t}}(x)=\phi(x)+\hat{\phi}(x)$ is the total porosity of the medium. By inverting (7.1), we obtain for $x \in \Omega$ a strictly monotone increasing function $\Sigma(x, \cdot) \in C^{0}([0,1])$ mapping onto $[0,1]$ such that

$$
s=\Sigma(x, S)
$$

We change variables according to (7.2). In the definition of weak solution, replace $\gamma_{1}(s), \gamma_{2}(s), f_{\mathrm{w}}(s), \lambda_{\mathrm{w}}(s)$, and $\lambda_{\mathrm{o}}(s)$ by $\left(\gamma_{1} \circ \Sigma\right)(S),\left(\gamma_{2} \circ \Sigma\right)(S)$, etc., respectively, and replace $\mathcal{S}(q)$ by $\left(\Sigma^{-1} \circ \mathcal{S}\right)(q)$. Finally, replace $\phi s+\hat{\phi} \sigma(s)$ by $\phi_{\mathrm{t}} S$ and $\phi s^{0}+$ $\hat{\phi} \sigma\left(s^{0}\right)$ by $\phi_{\mathrm{t}} S^{0}$, where $s^{0}=\Sigma\left(S^{0}\right)$. The resulting weak equations are those of an unfractured reservoir with saturation dependent functions modified by $\Sigma$ and with $\phi_{\mathbf{t}}$ replacing $\phi[17]$. Since (A1)-(A13) hold for the transformed system, we have our result.

\section{REFERENCES}

1. H. W. Alt and E. Di Benedetto, Nonsteady flow of water and oil through inhomogeneous porous media, Ann. Scuola Norm. Sup. Pisa Cl. Sci. (4) 12 (1985), 335-392.

2. H. W. Alt and S. Luckhaus, Quasilinear elliptic-parabolic differential equations, Math. Z. 183 (1983), 311-341.

3. S. N. Antoncev, On the solvability of boundary value problems for degenerate two-phase porous flow equations, Dinamika Splošnoŭ Sredy Vyp. 10 (1972), 28-53. (Russian)

4. S. N. Antoncev and V. N. Monahov, Three-dimensional problems of time-dependent two-phase filtration in nonhomogeneous anisotropic porous media, Soviet Math. Dokl. 19 (1978), 13541358.

5. T. Arbogast, A simplified dual-porosity model for two-phase flow, in "Proceedings of the Ninth International Conference on Computational Methods in Water Resources," Denver, 
Colorado, June 9-12, 1992, T. F. Russell, ed., Computational Mechanics Publications, Southampton, U.K. (to appear).

6. T. Arbogast, J. Douglas, Jr., and J. E. Santos, Two-phase immiscible flow in naturally fractured reservoirs, in "Numerical Simulation in Oil Recovery," Mary F. Wheeler, ed., The IMA Volumes in Mathematics and its Applications, Springer-Verlag, Berlin, New York, 1988, pp. $47-66$.

7. G. I. Barenblatt, Iu. P. Zheltov, and I. N. Kochina, Basic concepts in the theory of seepage of homogeneous liquids in fissured rocks [strata], Prikl. Mat. Mekh. 24 (1960), 852-864; J. Appl. Math. Mech. 24 (1960), 1286-1303.

8. J. Bear, "Dynamics of Fluids in Porous Media," Elsevier, New York, 1972.

9. N. T. Burdine, Relative permeability calculations from pore-size distribution data, Trans. Amer. Inst. Mining and Metallurgical Engrs. 198 (1953), 71-77.

10. G. Chavent, A new formulation of diphasic incompressible flows in porous media, in "Applications of Methods of Functional Analysis to Problems in Mechanics," Lecture Notes in Mathematics 503, P. Germain and B. Nayroles, eds., Springer-Verlag, Berlin, New York, 1976, pp. 258-270.

11. G. Chavent and J. Jaffré, "Mathematical Models and Finite Elements for Reservoir Simulation," North-Holland, Amsterdam, 1986.

12. Z. Chen and C. Liu, Theory of fluid displacement in a medium with double porosity, Energy Sources 6 (1982), 193-214.

13. R. E. Collins, "Flow of Fluids Through Porous Materials," Reinhold, New York, 1961.

14. J. Douglas, Jr. and T. Arbogast, Dual-porosity models for flow in naturally fractured reservoirs, in "Dynamics of Fluids in Hierarchical Porous Formations," J. H. Cushman, ed., Academic Press, London, 1990, pp. 177-221.

15. J. Douglas, Jr., T. Arbogast, and P. J. Paes Leme, Two models for the waterflooding of naturally fractured reservoirs, Paper SPE 18425, in "Proceedings, Tenth SPE Symposium on Reservoir Simulation," Society of Petroleum Engineers, Dallas, Texas, 1989, pp. 219-225.

16. J. Douglas, Jr., J. L. Hensley, and T. Arbogast, A dual-porosity model for waterflooding in naturally fractured reservoirs, Comp. Meth. in Appl. Mech. and Engng. 87 (1991), 157-174.

17. J. Douglas, Jr., P. J. Paes Leme, and J. L. Hensley, A limit form of the equations for immiscible displacement in a fractured reservoir, Transport in Porous Media (to appear).

18. J. R. Gilman, An efficient finite-difference method for simulating phase segregation in the matrix blocks in double-porosity reservoirs, Soc. Petroleum Engrs. J. 26 (July 1986), 403-413.

19. G. H. Hardy, J. E. Littlewood, and G. Pólya, "Inequalities," 2nd ed., Cambridge University Press, 1952.

20. H. Kazemi and J. R. Gilman, Improvements in simulation of naturally fractured reservoirs, Soc. Petroleum Engrs. J. 23 (Aug. 1983), 695-707.

21. D. Kroener and S. Luckhaus, Flow of oil and water in a porous medium, J. Differential Equations 55 (1984), 276-288.

22. S. N. Kružkov and S. M. Sukorjanskiř, Boundary problems for systems of equations of two-phase porous flow type; statement of the problems, questions of solvability, justification of approximate methods, Math. USSR Sbornik 33 (1977), 62-80.

23. M. C. Leverett, Capillary behavior in porous media, Trans. Amer. Inst. Mining and Metallurgical Engrs. 142 (1941), 341-358.

24. C. B. Morrey, Jr., "Multiple Integrals in the Calculus of Variations," Springer-Verlag, Berlin and New York, 1966.

25. D. W. Peaceman, "Fundamentals of Numerical Reservoir Simulation," Elsevier, New York, 1977.

26. A. E. Scheidegger, "The Physics of Flow Through Porous Media," 3rd ed., University of Toronto Press, Toronto, 1974. 
27. A. de Swaan, Theory of waterflooding in fractured reservoirs, Soc. Petroleum Engrs. J. 18 (April 1978), 117-122.

28. J. E. Warren and P. J. Root, The behavior of naturally fractured reservoirs, Soc. Petroleum Engrs. J. 3 (Sept. 1963), 245-255. 